\title{
The Active Suspension of a Cab in a Heavy Machine
}

\author{
Grzegorz Tora
}

Additional information is available at the end of the chapter

http://dx.doi.org/10.5772/45831

\section{Introduction}

The work of operators in heavy machinery requires constant attention to gather information about the machine's surroundings, its current status and the operations performed. Operators have to analyse the received information on the continuous basis and make decisions accordingly, to have them implemented via the control system and to perform the scheduled tasks in the optimal manner. The more powerful the machine, the more serious the consequence of errors committed by operators. The typical frequency range of vibration of machines and their equipment is determined based on testing done on heavy machines used in Europe [1] and is found to be $0.5-80 \mathrm{~Hz}$.

Machine vibrations are induced by the drives' action, movements of the equipment, variable loading and machine ride. The ride of heavy machines, tractors, forestry vehicles over a rough terrain lead to cyclic tilting of the machines, which can be regarded as low-frequency (up to several $\mathrm{Hz}$ ) and high-amplitude (about 10 degrees) vibration of the machine. The angular motions of the frame are transmitted onto the cab, and the higher the cab position, the larger the amplitude range of linear vibration of the point SIP (about $70 \mathrm{~cm}$ ). Vibrations negatively impact on the machine structure, control processes, performance quality and the operator's comfort. Growing ergonomic concerns and competition on the market have prompted the design of machines ensuring the better comfort for the operator.

Cab suspensions are now incorporated in the machine structure as a new solution. The active suspension is a system whose components are based on existing vibration reduction solutions. Early vehicles were also provided with suspension systems to suppress vibrations due to the ride in the rough terrain. At first these were passive suspension systems, in which the characteristics of the components i.e. elastic and damping elements are fixed. Suspensions incorporating semiactive elements perform better as vibration isolation systems since their characteristics can be varied according to the adopted control strategy. Active suspensions 
lend a new quality to control of low-frequency vibrations in vehicles. The operating ranges of passive, semiactive and active suspensions, given as velocity-force characteristics, are shown in Fig 1.

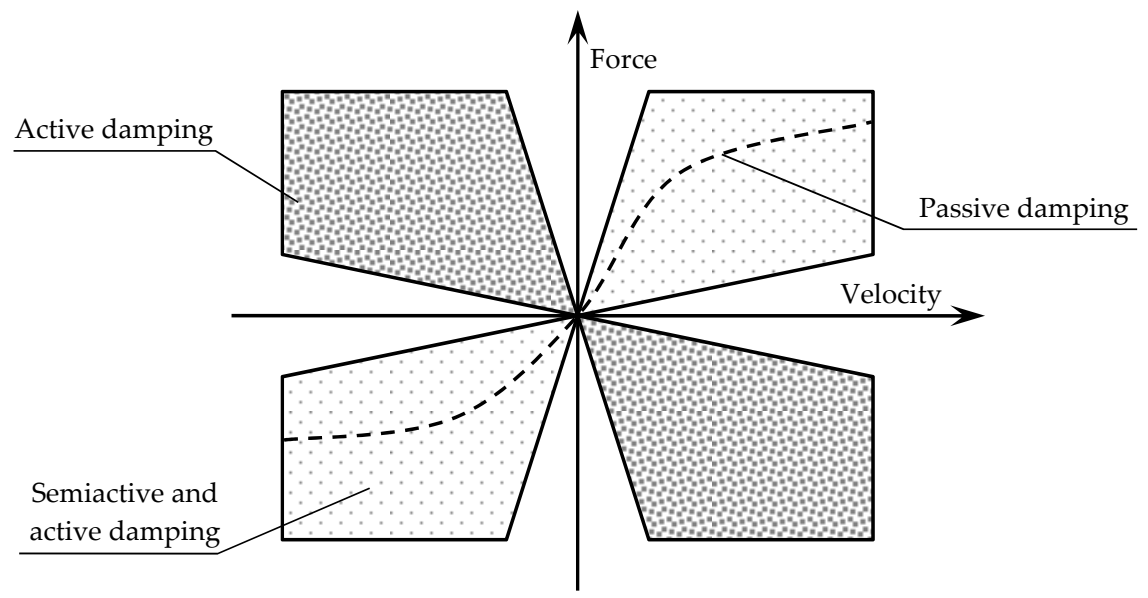

Figure 1. The range of velocity-force characteristics of passive, semiactive and active suspensions [2]

Passive suspensions are described with a damper characteristics with fixed parameters (broke line). These suspensions typically comprise elastic elements featuring a linear or nonlinear elasticity and damping elements with nonlinear characteristics. On one hand, stability of parameters of a passive suspension system is considered an advantage as its construction can be made simple, but on the other hand the vehicle suspension will not perform optimally in response to inputs other than average. Steel and rubber connectors used as joint components in vehicle suspensions play a major role in damping higher-frequency vibrations.

The areas in the first and third quadrant have relevance to the family of semiactive characteristics. A semiactive element comprises a damping element whose damping ratio can be varied through the real-time control process. A semiactive suspension does not generate any active force, hence the power demand remains on a low level. The damping ratio can be varied using throttling valves or through application of rheological fluids whose stiffness and viscosity depend on electric or magnetic field intensity. The work [3] investigates the potential applications of dry friction in semiactive dampers. Another solution uses a lever system wherein the attachment point of the spring can be varied and its elasticity controllable [4]. The upper frequency limit for the effective performance of semiactive suspension systems is about $100 \mathrm{~Hz}$. Because of their low power demand, semiactive suspensions systems are being vigorously researched and widely implemented in vehicles.

The operation of active suspension systems is revealed in characteristics in all four quadrants, whereas the second and fourth quadrant capture the conditions where the actuator requires an external energy source, otherwise it acts as a passive element, dissipating energy. Active suspension systems incorporate a force actuator, either independent, or connected in parallel to a damper or a spring. Active suspensions prove 
most effective in the case of low-frequency vibrations [5], about $5 \mathrm{~Hz}$ and are often added to passive systems which well handle high-frequency vibrations. High efficiency of active systems, however, comes at the cost of high power demand. Active suspension systems typically utilise small hydraulic cylinders to achieve high accelerations of masses subjected to considerable loads. Pneumatic cylinders are capable of delivering higher velocity than hydraulic ones, yet the acceleration is strongly dependent on the external load applied. There are also hydro-pneumatic cylinders, displaying the advantages of the two previous types. Active suspension systems in truck cabs utilise electric cylinders DC and AC-servo.

The new developments of the operator's seats are in line with the advancements in vehicle suspensions. In the simplest solution where the seat is fixed rigid to the machine frame or to the cab floor, vibrations are transmitted from the cab attachment point onto the operator. The mobile seat support allows it to be moved only in the vertical so the passive, semiactive or active vibration reduction system can be added along this direction only. Typically, a shearing mechanism is used as seat support [6], though other solutions with a greater number of DOFs are reported as well [7]. On account of small mass of the seat together with the operator, the upper frequency limit is reached and the active support becomes more effective than a vehicle suspension. Active suspension systems are now incorporated in agricultural and forestry machinery, where typical excitations are in the form of lowfrequency and high-amplitude vibrations [8].

The correct control strategy is a key element in active and semiactive vibration reduction systems, resulting in a good compromise between numerous and sometimes mutually excluding requirements. Optimal control techniques that handle this problem include the linear quadratic regulator LQR [9] and the linear Gaussian regulator LQG [10]. In simpler cases the PID control can be applied. Active control systems may also use regulators based on neural networks [11] and fuzzy logics [12]. The control schemes that are commonly used for semiactive suspension systems include the 'sky-hook' control strategy [13] where the damping force is related to the absolute velocity of the vehicle body. The $\mathcal{H}_{\infty}$ control is insensitive to uncertain input quantities, encumbered with major errors (for instant: time constant, reduced mass, damping force, acceleration, velocity, damping ratio for the tire [14]. The $\mathcal{H}_{\infty}$ control scheme concurrently executes the mutlicriterial optimisation and in active and semiactive suspension systems it takes into account the acceleration of sprung mass, peak accelerations, jerks of the front and rear suspension, road holding, forces acting upon the relevant masses, deflection of the tire and of the suspension [15]. The work [16] focused on $\mathcal{H}_{\infty}$ control in an active suspension of vehicle investigates the influence of the time delay on stability of the control process. It is shown that the delay time (i.e. time before the cylinder in the active system is activated), the reduced mass and power ratings are major determinants of the frequency limit for effective operation of these type of suspensions. The stability condition is formulated for the predetermined range of time delay and system parameters. Extensive expertise prompts the use of filters, beside robust control for the purpose of estimation. The Kalman filter, reported in literature on the subject [17], is now widely used.

To improve the operator's comfort, an active suspension of a cab can be incorporated in the machine structure (Fig 2), to reduce the cab's vibration. The active suspension system comprises several sub-systems: 


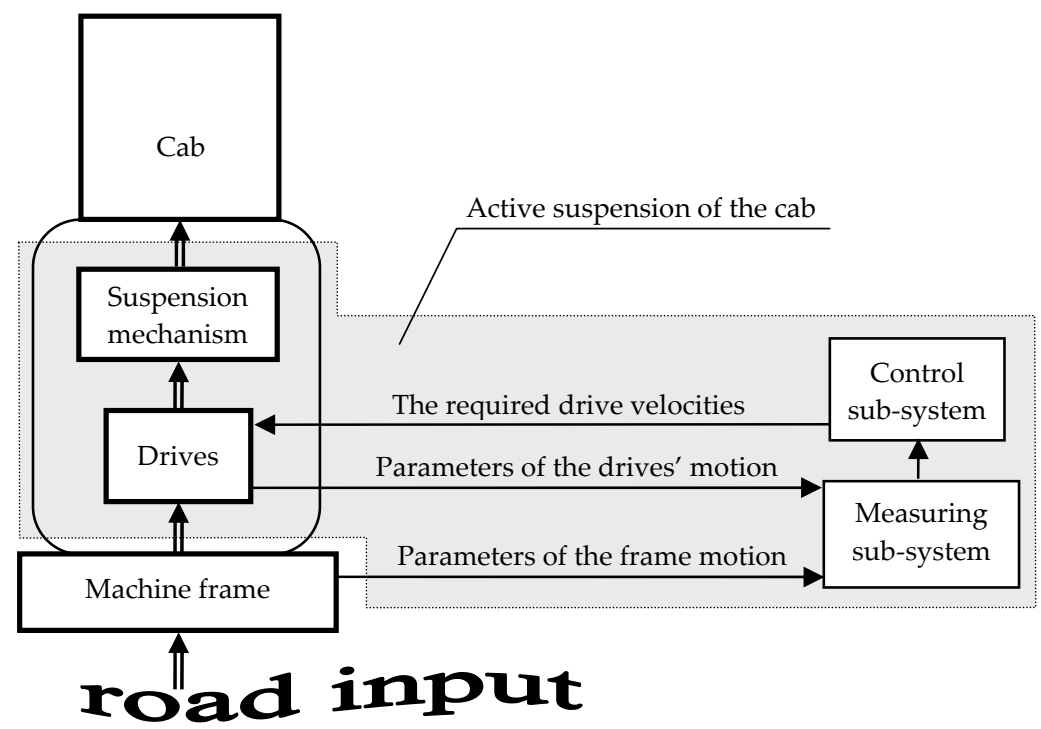

Figure 2. Block diagram of the active suspension of a cab

1. The actuator mechanism, connected to the machine frame and the cab is placed in between. The main element (link) of the mechanism is a mobile platform to which the cab is attached. The platform is suspended or supported on the frame and depending on the mechanism's mobility, it can move with respect to the frame in the selected DOFs.

2. The drives set in motion the passive links in the active suspension mechanism. On account of the stiffness requirements and availability of the given type of energy, and to ensure fast response the control signals the hydraulic drives are going to be used. The drives are provided with actuators to capture the instantaneous velocities, derived in the control sub-system.

3. Measuring sub-system. Displacement and velocity are chosen as control quantities for the active suspension system. Directly measured data yield the error signal to be used in the control process. The machine frame subjected to kinematic excitations executes a spatial movement, measured with a set of sensors.

4. Control sub-system. Errors of drive positions and their derivatives are going to be used in the feedback control of the active suspension system. Basing on the frame motion measurements, the control sub-system performs the real time calculation of the anticipated loads and the required drive velocities.

\section{Actuator mechanism in the active suspension system}

The active suspension mechanism, shown schematically in Fig 3 has been engineered specifically for the purpose of modelling and simulations and its design involves a certain trade-off between functionality and simplicity. The presented active suspension mechanism is capable of reducing the amplitudes of the cab's linear vibrations in the direction $y_{r}$ and its angular vibrations around the axes $x_{r}, y_{r}$. The active suspension mechanism comprises just 
three passive links, set in motion by two linear drives. The separate seat suspension mechanism reduces the vibrations along the axis $z_{r}$. The main function of the active suspension system is to stabilise the cab such that the correct control of the drives 1 and 4 should enable its vertical movement in the direction of the gravity force. The active suspension system comprises a platform $p$ suspended on three limbs with spherical pairs having the centres $B_{1}, B_{2}, B_{3}$. The two limbs are rocker arms 2 and 3 , connected to the machine frame $r$ via a revolving pair. The third limb is the actuator 1 with the length $s_{1}$. The cylinder in the actuator $1 c$ is connected to the frame $r$ via a cross pair with the point $A_{1}$. The piston in the actuator $4 t$ is connected to the rocker arm via a spherical joint with the point $C_{4}$. The length of the actuator 4 equals 54 . The part of the active suspension system comprising the rocker arms 2 and 3 , a actuator and the platform $p$ along the line segment $B_{2} B_{3}$ can be treated as a planar mechanism where the points $A_{4}, C_{4}, A_{2}, B_{2}$ and $A_{3}$ are on the plane $y_{r} z_{r}$ and the axes of joints $A_{2}$ and $A_{3}$ are parallel to $x_{r}$. The structure of the mechanism is such that the actuators 1 and 4, when in their middle position, do not carry the cab's gravity load and when in their extreme positions, the load due to the gravity force is carried mostly by the joints $A_{2}$, $A_{3}$. Besides, the performance of the mechanism is affected by manufacturing imprecision, though this influence is found to be negligible.

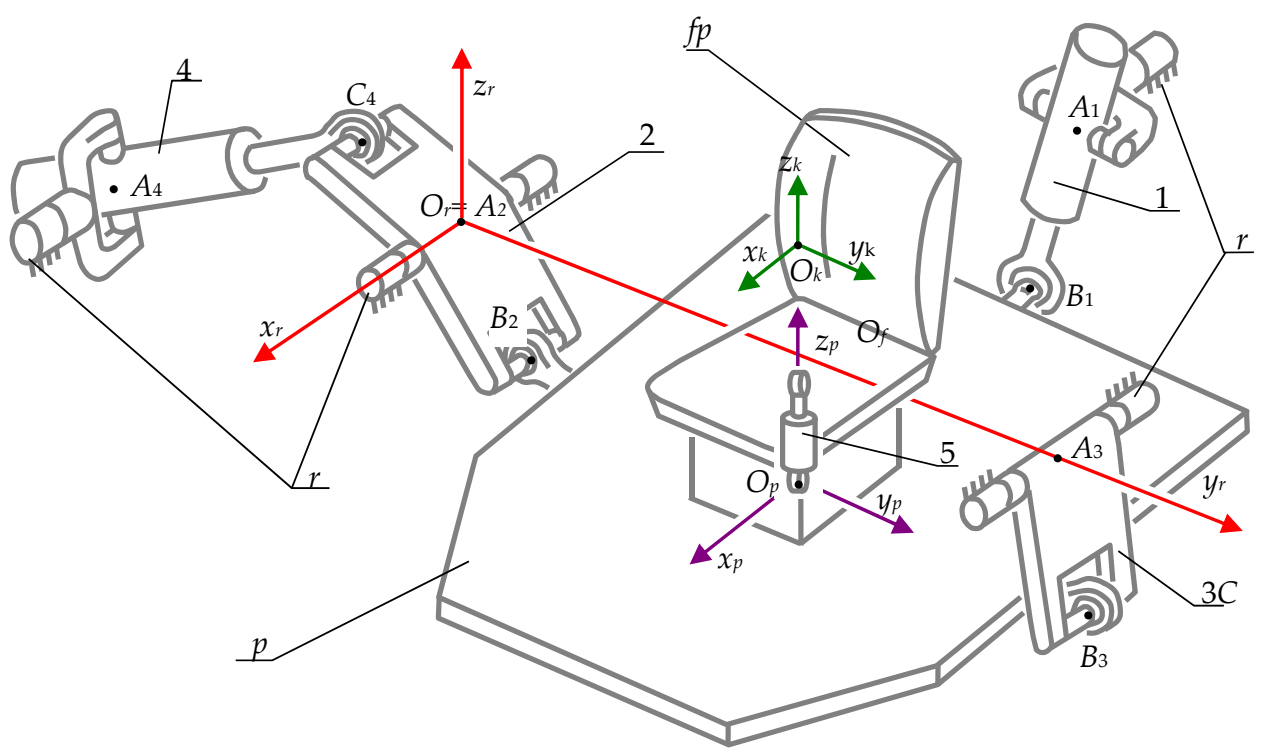

Figure 3. Platform mechanism stabilising the cab in the vertical: $r$ - machine frame, $p$ - platform, 2,3 rocker arms, $4 w$ - forks in cross joints, $1 c, 4 c$ - cylinders in actuators, $1 t, 4 t$ - pistons in cylinders

The cab is rigidly attached to the platform $p$. The centre of gravity (c.o.g) of the cab is at the point $Q_{k}$. The reference systems associated with the platform $\left\{Q_{p} x_{p} y_{p} z_{p}\right\}$ and with the cab $\left\{Q_{k} x_{k} y_{k} z_{k}\right\}$ are parallel and immobile with respect to one another. Inside the cab there is a movable operator's seat $f$, which can be moved with respect to the cab, along its vertical axis $z k$. The seat suspension mechanism with the operator is not the subject matter of the present 
study. The centre of gravity of the seat and the operator is at the point $Q_{f}$, whose vertical coordinate in the reference system associated with the platform is controlled by the drive 5 , implementing seat elevation.

\section{Model of the kinematic excitation of the machine frame motion}

To implement the control, we need to know the angles of deflection of the vertical frame axis in the direction of the gravity force (Fig 4). The first measured angle defines the frame rotation around the longitudinal axis $\alpha_{x}$, the other angle defines the frame rotation round the lateral axis $\alpha_{y}$.

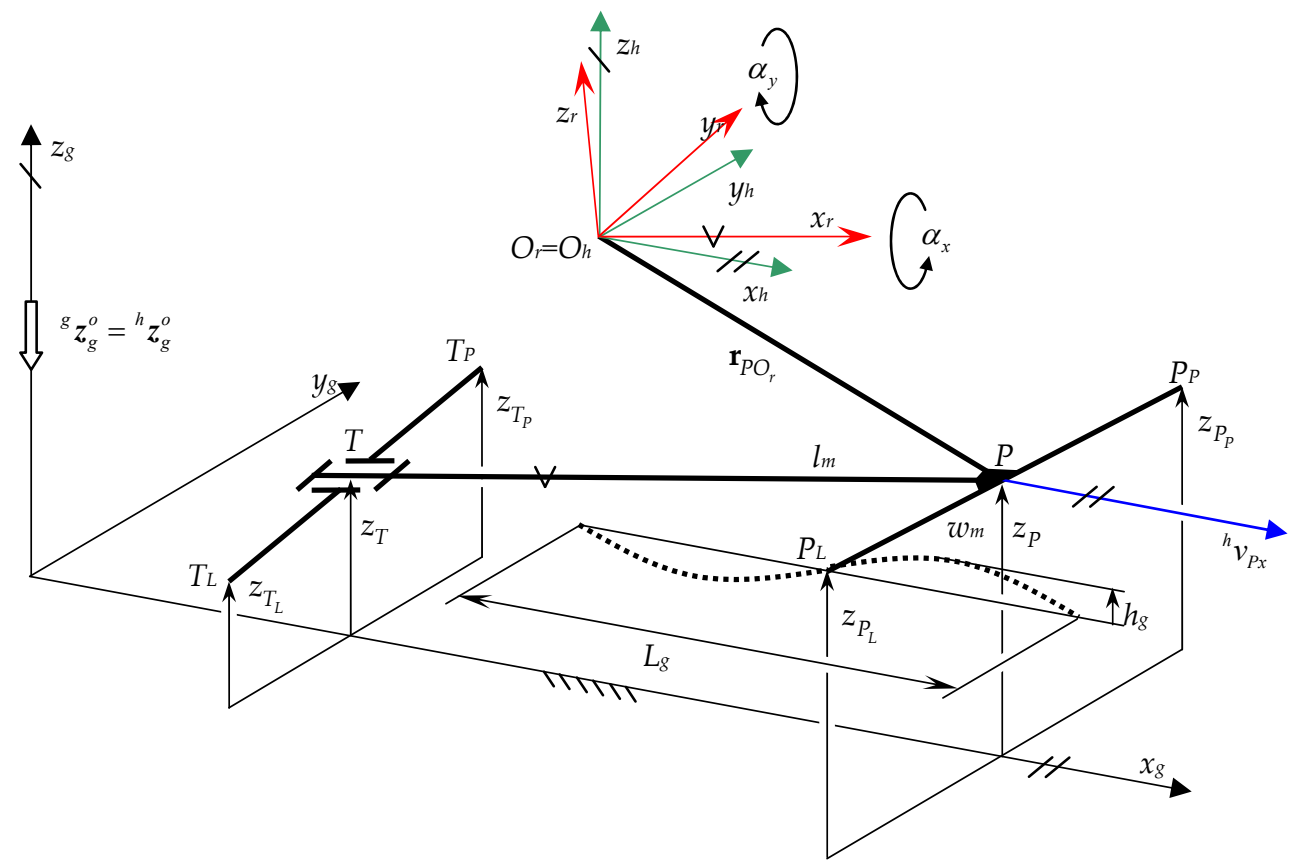

Figure 4. Model of the kinematic excitation inducing the motion of the machine frame

The model of the active suspension mechanism uses several reference systems. The immobile system $\left\{Q_{8} x_{8} y_{8} z_{g}\right\}$ is associated with the road travelled by the machine. This system is used to define the function of road profile on which the machine travels. The mobile system $\left\{Q_{r} x_{r} y_{r} z_{r}\right\}$ is associated with the machine frame (Fig 3, Fig 4). Its origin $O_{r}=A_{2}$ is one of the attachments points of the active suspension mechanism to the frame. This system is recalled to define all kinematic and dynamic quantities (no superscript on the left). The

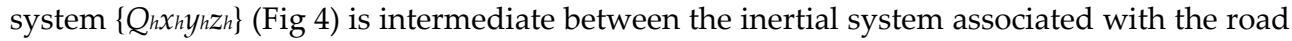
and the mobile system associated with the frame. The origins of the reference systems $\left\{Q_{h} x_{h} y_{h} z_{h}\right\}$ and $\left\{Q_{r} x_{r} y_{r} z_{r}\right\}$ will coincide: $O_{h}=O_{r}$. The axes $z_{h}$ and $z_{g}$ are parallel and the plane determined by $x_{h} z h$ contains the axis $x_{r}$. In the model of the active suspension, the directional 
versor of the gravity force ${ }^{h} \mathbf{z}_{g}^{o}=[0,0,-1]^{T}$ given in the reference system $\left\{Q_{h} x_{h} y h z h\right\}$ should be expressed in the system $\left\{Q_{r} x_{r} y_{r} z_{r}\right\}$ :

$$
\mathbf{z}_{g}^{o}={ }_{h}^{r} \mathbf{R}^{h} \mathbf{z}_{g}^{o}
$$

where: ${ }_{h}^{r} \mathbf{R}\left(\alpha_{x}, \alpha_{y}\right)=\left[\begin{array}{ccc}\cos \alpha_{y} & 0 & \sin \alpha_{y} \\ \sin \alpha_{x} \sin \alpha_{y} & \cos \alpha_{x} & -\sin \alpha_{x} \cos \alpha_{y} \\ -\cos \alpha_{x} \sin \alpha_{y} & \sin \alpha_{x} & \cos \alpha_{x} \cos \alpha_{y}\end{array}\right]$ - the transition matrix from the system $\left\{Q_{h} x_{h} y_{h} z h\right\}$ to $\left\{Q_{r} x_{r} y_{r} z_{r}\right\}$, derived basing on the frame deflection angles $\alpha_{x}, \alpha_{y}$ measured in the measuring sub-system.

Underlying the simulation procedure is the model of the kinematic excitation applied to the machine frame, shown in Fig 4.

The machine frame is represented by a front bridge $\left(P_{P}, P_{T}\right)$, a longitudinal frame $(P, T)$ and a joint at the point $O_{r}$, where the active suspension mechanism is connected to the frame. The rear bridge $\left(T_{L}, T_{P}\right)$ is connected to the longitudinal frame via a revolving pair $T$. It is assumed that:

- $\quad$ the longitudinal axis of the frame TP moves in the plane $x_{g} z_{g}$,

- $\quad$ velocity components of the extreme points of the front and rear bridge along the axis $x_{g}$ are identical and equal to ${ }^{h} v_{P x}$, whilst their velocity components in the direction of $y_{g}$ are negligible,

- velocity components in the direction $z_{g}$ are associated with vertical displacements of the wheels during the ride in rough terrain.

The vertical displacement of the centre of the front right wheel is governed by a harmonic function:

$$
z_{P_{P}}=h_{o}+h_{g}\left[1-\cos \left(2 \pi \frac{{ }^{h} v_{P x} t}{L_{g}}\right)\right]
$$

When $t<t_{\varphi}$, then vertical displacement of the centre of the front left wheel is taken to be $z_{P_{L}}=h_{o}$. When $t \geq t_{\varphi}$, vertical displacement of the centre of the front left wheel is expressed as the harmonic:

$$
z_{P_{L}}=h_{o}+h_{g}\left[1-\cos \left(2 \pi \frac{h_{v_{P x}}\left(t-t_{\varphi}\right)}{L_{g}}\right)\right]
$$

When $t<t_{l_{m}}$, the vertical displacement of the centre of the rear right wheel is taken to be $z_{T_{p}}=h_{o}$. When $t \geq t_{l_{m}}$, the vertical displacement is expressed as harmonic:

$$
z_{T_{P}}=h_{o}+h_{g}\left[1-\cos \left(2 \pi \frac{{ }^{h} v_{P x}\left(t-t_{l_{m}}\right)}{L_{g}}\right)\right]
$$


When $t<t_{l_{m}}+t_{\varphi}$, the vertical displacement of the centre of the rear left wheel is taken to be $z_{T_{L}}=h_{o}$. When $t \geq t_{l_{m}}+t_{\varphi}$, the vertical displacement is expressed as harmonic:

$$
z_{T_{L}}=h_{o}+h_{g}\left[1-\cos \left(2 \pi \frac{{ }^{h} v_{P x}\left(t-t_{l_{m}}-t_{\varphi}\right)}{L_{g}}\right)\right]
$$

where: $t_{\varphi}=\frac{\varphi L_{g}}{2 \pi^{h} v_{P x}}-$ phase shift time between the left and right hand side of the machine,

$t_{l_{m}}=\frac{l_{m}}{v_{P x}}$ - phase shift time between the front and rear part of the machine,

$L_{g}$ - distance corresponding to the full wave, $h_{g}$ - amplitude, $w_{m}$ - width of the front and rear bridge,

$l_{m}$-distance between the front and rear bridge, $\varphi$ - phase shift angle of the road profile between the left and right-hand side of the machine.

\section{Kinematic model of the active suspension mechanism}

To determine the influence of the active suspension system on the cab motion, the kinematic model is developed based on vector calculus. Versors used to define the positions of the active suspension mechanism links are shown in Fig 5.

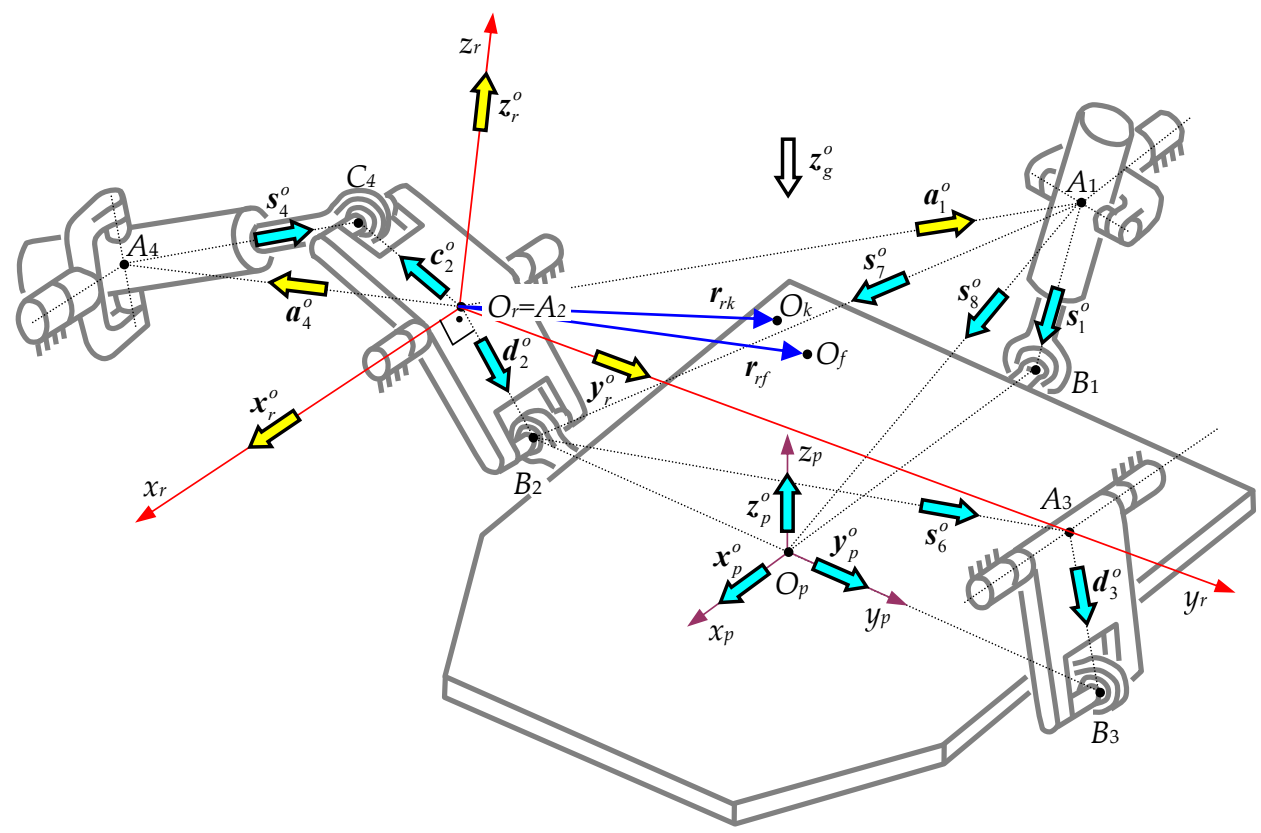

Figure 5. Versors in the kinematic model of cab stabilisation in the vertical 


\subsection{Direct kinematics problem of links position}

Solving the direct problem consists in finding the cab orientation and position of its centre of gravity $Q_{k}$ and of the point $Q_{f}$ - the centre of gravity of the seat-operator system with respect to the reference system associated with the machine frame. The cab orientation is determined by directional versors in the reference system associated with the platform $\mathbf{x}_{p}^{o}, \mathbf{y}_{p}^{o}, \mathbf{z}_{p}^{o}$. The cab position (and of the platform and the seat) and $\mathbf{r}_{r k}$ - the radius vector of the of the cab's c.o.g with respect to the reference system associated with the frame depend on variable lengths of actuators $s_{1}, s_{4}$ and the known constant dimensions of links in the active suspension mechanism. The radius vector of the c.o.g of the seat-operator system; $\mathbf{r}_{r f}$ is controlled by the lengths of three actuators $s_{1}, s_{4}, s_{5}$ and the fixed dimensions of links in the active suspension mechanism. The solution of the simple problem involving the link position is explicit and consists in determining versors on the basis of two already known or already established ones.

The versor $\mathbf{c}_{2}^{o}$ is derived basing on two known versors $\mathbf{a}_{4}^{o}=\left[0, a_{4 y}^{o}, a_{4 z}^{o}\right]^{T}$ and $\mathbf{x}_{r}^{o}=[1,0,0]^{T}$ :

$$
\mathbf{c}_{2}^{o}=\mathbf{a}_{4}^{o} \frac{c_{2}^{2}-s_{4}^{2}+a_{4}^{2}}{2 a_{4} c_{2}}+\left(\mathbf{a}_{4}^{o} \times \mathbf{x}_{r}^{o}\right) \sqrt{1-\left(\frac{c_{2}^{2}-s_{4}^{2}+a_{4}^{2}}{2 a_{4} c_{2}}\right)^{2}}
$$

where: : $s_{4}=A_{4} C_{4}, c_{2}=O_{r} C_{4}, a_{4}=O_{r} A_{4}$.

The versor $\mathbf{s}_{4}^{o}$ is obtained basing on $\mathbf{a}_{4}^{o}$ and $\mathbf{c}_{2}^{o}$ :

$$
\mathbf{s}_{4}^{o}=-\frac{a_{4}}{s_{4}} \mathbf{a}_{4}^{o}+\frac{c_{2}}{s_{4}} \mathbf{c}_{2}^{o}
$$

The versor $\mathbf{d}_{2}^{o}$ is obtained basing on $\mathbf{x}_{r}^{o}$ and $\mathbf{c}_{2}^{o}$ :

$$
\mathbf{d}_{2}^{o}=c_{22} \mathbf{c}_{2}^{o}+\sqrt{1-c_{22}^{2}}\left(\mathbf{x}_{r}^{o} \times \mathbf{c}_{2}^{o}\right)
$$

where: $c_{22}=\mathbf{c}_{2}^{o} \cdot \mathbf{d}_{2}^{o}=\cos \measuredangle\left(\mathbf{c}_{2}^{o}, \mathbf{d}_{2}^{o}\right), \measuredangle\left(\mathbf{c}_{2}^{o}, \mathbf{d}_{2}^{o}\right)$ - a known fixed angle.

The versor $\mathbf{s}_{6}^{o}$ is obtained basing on $\mathbf{d}_{2}^{o}$ and $\mathbf{y}_{r}^{o}=[0,1,0]^{T}$ :

$$
\mathbf{s}_{6}^{o}=-\frac{d_{2}}{s_{6}} \mathbf{d}_{2}^{o}+\frac{a_{3}}{s_{6}} \mathbf{y}_{r}^{o}
$$

where: $s_{6}=\sqrt{d_{2}^{2}+a_{3}^{2}-2 d_{2} a_{3}\left(\mathbf{d}_{2}^{o} \cdot \mathbf{a}_{3}^{o}\right)}, d_{2}=O_{r} B_{2}, a_{3}=O_{r} A_{3}$.

The versor $\mathbf{d}_{3}^{o}$ is obtained basing on $\mathbf{s}_{6}^{o}$ i and $\mathbf{x}_{r}^{o}$ :

$$
\mathbf{d}_{3}^{o}=\mathbf{s}_{6}^{o} \frac{d_{3}^{2}-b_{23}^{2}+s_{6}^{2}}{2 s_{6} d_{3}}-\mathbf{s}_{6}^{o} \times \mathbf{x}_{r}^{o} \sqrt{1-\left(\frac{d_{3}^{2}-b_{23}^{2}+s_{6}^{2}}{2 s_{6} d_{3}}\right)^{2}}
$$


where: $d_{3}=A_{3} B_{3}, b_{23}=B_{2} B_{3}$.

The versor $\mathbf{y}_{p}^{o}$ is obtained basing on $\mathbf{s}_{6}^{o}$ and $\mathbf{d}_{3}^{o}$ :

$$
\mathbf{y}_{p}^{o}=\frac{s_{6}}{b_{23}} \mathbf{s}_{6}^{o}+\frac{d_{3}}{b_{23}} \mathbf{d}_{3}^{o}
$$

The versor $\mathbf{s}_{7}^{o}$ is obtained basing on $\mathbf{d}_{2}^{o}$ and $\mathbf{a}_{1}^{o}=\left[a_{1 x}^{o}, a_{1 y}^{o}, a_{1 z}^{o}\right]^{T}$ :

$$
\mathbf{s}_{7}^{o}=-\frac{d_{2}}{s_{7}} \mathbf{d}_{2}^{o}+\frac{a_{1}}{s_{7}} \mathbf{a}_{1}^{o}
$$

where: $s_{7}=\sqrt{d_{2}^{2}+a_{1}^{2}-2 d_{2} a_{1}\left(\mathbf{d}_{2}^{o} \cdot \mathbf{a}_{1}^{o}\right)}, a_{1}=O_{r} A_{1}$.

The versor $\mathbf{s}_{8}^{o}$ is obtained basing on $\mathbf{s}_{7}^{o}$ and $\mathbf{y}_{p}^{o}$ :

$$
\mathbf{s}_{8}^{o}=\frac{s_{7}}{s_{8}} \mathbf{s}_{7}^{o}+\frac{b_{2}}{s_{8}} \mathbf{y}_{p}^{o}
$$

where: $s_{8}=\sqrt{s_{7}^{2}+b_{2}^{2}-2 s_{7} b_{2}\left(\mathbf{s}_{7}^{o} \cdot \mathbf{y}_{p}^{o}\right)}, b_{2}=O_{p} B_{2}$.

The versor $\mathbf{s}_{1}^{o}$ is obtained basing on $\mathbf{s}_{7}^{o}$ and $\mathbf{s}_{8}^{o}$ :

$$
\mathbf{s}_{1}^{o}=\frac{c_{71}-c_{78} c_{81}}{1-c_{78}^{2}} \mathbf{s}_{7}^{o}+\frac{c_{81}-c_{78} c_{71}}{1-c_{78}^{2}} \mathbf{s}_{8}^{o}-\frac{\sqrt{1-c_{78}^{2}-c_{71}^{2}-c_{81}^{2}+2 c_{78} c_{71} c_{81}}}{1-c_{78}^{2}}\left(\mathbf{s}_{7}^{o} \times \mathbf{s}_{8}^{o}\right)
$$

where: $c_{71}=\frac{s_{7}^{2}+s_{1}^{2}-b_{12}^{2}}{2 s_{1} s_{7}}, c_{78}=\frac{s_{7}^{2}+s_{8}^{2}-b_{2}^{2}}{2 s_{7} s_{8}}, c_{81}=\frac{s_{8}^{2}+s_{1}^{2}-b_{1}^{2}}{2 s_{1} s_{8}}, b_{1}=O_{p} B_{1}, b_{12}=B_{1} B_{2}$.

The versor $\mathbf{x}_{p}^{o}$ is obtained basing on $\mathbf{s}_{1}^{o}$ and $\mathbf{s}_{8}^{o}$ :

$$
\mathbf{x}_{p}^{o}=-\frac{s_{1}}{b_{1}} \mathbf{s}_{1}^{o}+\frac{s_{8}}{b_{1}} \mathbf{s}_{8}^{o}
$$

where: $b_{1}=O_{p} B_{1}$.

The versor $\mathbf{z}_{p}^{o}$ is obtained basing on $\mathbf{x}_{p}^{o}$ and $\mathbf{y}_{p}^{o}$ :

$$
\mathbf{z}_{p}^{o}=\mathbf{x}_{p}^{o} \times \mathbf{y}_{p}^{o}
$$

Eq (11), (15) and (16) yield the versors of the platform $p$, and the matrix ${ }_{p}^{r} \mathbf{R}$ - the direction matrix of the reference system associated with the platform with respect to the system associated with the frame: 


$$
{ }_{p}^{r} \mathbf{R}=\left[\begin{array}{ccc}
\mathbf{x}_{p}^{o} \cdot \mathbf{x}_{r}^{o} & \mathbf{y}_{p}^{o} \cdot \mathbf{x}_{r}^{o} & \mathbf{z}_{p}^{o} \cdot \mathbf{x}_{r}^{o} \\
\mathbf{x}_{p}^{o} \cdot \mathbf{y}_{r}^{o} & \mathbf{y}_{p}^{o} \cdot \mathbf{y}_{r}^{o} & \mathbf{z}_{p}^{o} \cdot \mathbf{y}_{r}^{o} \\
\mathbf{x}_{p}^{o} \cdot \mathbf{z}_{r}^{o} & \mathbf{y}_{p}^{o} \cdot \mathbf{z}_{r}^{o} & \mathbf{z}_{p}^{o} \cdot \mathbf{z}_{r}^{o}
\end{array}\right]
$$

The solution to the simple problem involving the link position is complete when the positions of points $O_{k}$. and $O_{f}$ are found in relation to $s_{1}, s_{4}, s_{5}$. The radius vector from the origin of the reference system associated with the frame $O_{r}$ to the point $O_{f}$ becomes (Fig 5):

$$
\mathbf{r}_{r f}=d_{2} \mathbf{d}_{2}^{o}+b_{2} \mathbf{y}_{p}^{o}+{ }_{p}^{r} \mathbf{R}^{p} \mathbf{r}_{p f}
$$

where: ${ }^{p}\left(\overline{O_{p} O_{f}}\right)={ }^{p} \mathbf{r}_{p f}=\left[\begin{array}{lll}{ }^{p} r_{(p f) x} & { }^{p} r_{(p f) y} & { }^{p} r_{(p f) z}\end{array}\right]^{T}$ - radius vector of the point $O_{r}$ in the system associated with the platform, ${ }^{p} r_{(p f) z}=d f_{z}+s_{5}$ - variable vertical coordinate of the seat controlled by the seat elevating drive 5. ${ }^{p} r_{(p f) x},{ }^{p} r_{(p f) y}, d f_{z}$ - fixed coordinates.

The radius vector from the origin of the reference system associated with the frame $O_{r}$ to the point $O_{k}$ becomes:

$$
\mathbf{r}_{r k}=d_{2} \mathbf{d}_{2}^{o}+b_{2} \mathbf{y}_{p}^{o}+{ }_{p}^{r} \mathbf{R}^{p} \mathbf{r}_{p k}
$$

where: ${ }^{p}\left(\overline{O_{p} O_{k}}\right)={ }^{p} \mathbf{r}_{p k}=\left[\begin{array}{lll}{ }^{p} r_{(p k) x} & { }^{p} r_{(p k) y} & { }^{p} r_{(p k) z}\end{array}\right]^{T}$ - radius vector of the point $O_{k}$ in the system associated with the platform. ${ }^{p} r_{(p k) x},{ }^{p} r_{(p k) y},{ }^{p} r_{(p k) z}$ - fixed coordinates

\subsection{Inverse kinematics problem of link position}

The inverse problem handled in the coordinate system associated with the machine frame involves the orientation of the platform $p$. The platform should be stabilised in the vertical whilst the active suspension system is in use. The platform position is related to the gravity force versor $\mathbf{z}_{g}^{o}($ Fig $4,5,6)$, which can be expressed in the coordinate system associated with the frame according to the formula (1).

In order to solve the inverse problem it is required that the lengths of the actuators $s 10, s_{4}$ should be established, corresponding to the predetermined and expected platform position with respect to the system associated with the frame and expressed by versor coordinates: $\mathbf{x}_{p_{0}}^{o}, \mathbf{y}_{p_{0}}^{o}$ and $\mathbf{z}_{p o}^{o}$. Actually, the cab will reach the position nearing the expected one. The anticipated values (indicated with a subscript " ${ }_{0}$ ") obtained from solving the inverse problem will be used to derive the error signal required for the control process. The direction of the cab's vertical axis versor should be opposite to that of the gravity force versor $\mathbf{z}_{p o}^{o}=-\mathbf{z}_{g}^{o}$.

The versor $\mathbf{y}_{p o}^{o}$ is obtained basing on $\mathbf{z}_{p o}^{o}$ and $\mathbf{x}_{r}^{o}$ :

$$
\mathbf{y}_{p o}^{o}=\mathbf{z}_{p o}^{o} \times \mathbf{x}_{r}^{o}
$$




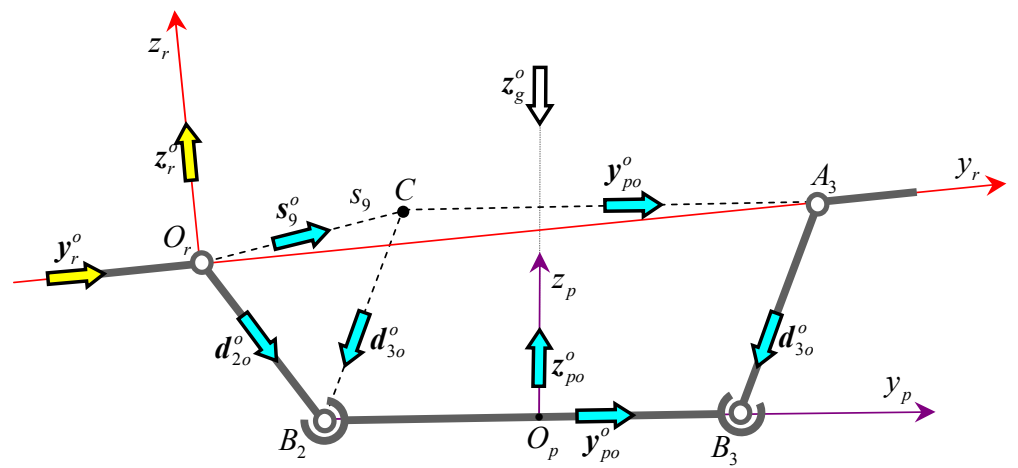

Figure 6. Solving the inverse problem- schematic diagram

The versor $\mathbf{x}_{p o}^{o}$ is obtained basing on $\mathbf{y}_{p o}^{o}$ and $\mathbf{z}_{p o}^{o}$ :

$$
\mathbf{x}_{p o}^{o}=\mathbf{y}_{p o}^{o} \times \mathbf{z}_{p o}^{o}
$$

The modulus of the vector between points $O_{r}$ and $C$ and its versor are computed using the triangle $\mathrm{O}_{r} A_{3} C$ (Fig 6):

$$
\begin{gathered}
s_{9}=\sqrt{a_{23}^{2}+b_{23}^{2}-2 a_{23} b_{23}\left(\mathbf{y}_{p o}^{o} \cdot \mathbf{y}_{r}^{o}\right)} \\
\mathbf{s}_{9}^{o}=\frac{a_{23} \mathbf{y}_{r}^{o}-b_{23} \mathbf{y}_{p o}^{o}}{s_{9}}
\end{gathered}
$$

The versor $\mathbf{d}_{2 o}^{o}$ is obtained basing on $\mathbf{s}_{9}^{o}, s_{9}, d_{2}$ and $d_{3}$ :

$$
\mathbf{d}_{2 o}^{o}=-\sqrt{1-\left(\frac{d_{2}^{2}-d_{3}^{2}+s_{9}^{2}}{2 s_{9} d_{2}}\right)^{2}} \mathbf{x}_{r}^{o} \times \mathbf{s}_{9}^{o}+\frac{d_{2}^{2}-d_{3}^{2}+s_{9}^{2}}{2 s_{9} d_{2}} \mathbf{s}_{9}^{o}
$$

The versor $\mathbf{c}_{20}^{o}$ is obtained basing on $\mathbf{x}_{r}^{o}$ and $\mathbf{d}_{20}^{o}$ :

$$
\mathbf{c}_{2 o}^{o}=c_{22} \mathbf{d}_{2 o}^{o}-\sqrt{1-c_{22}^{2}}\left(\mathbf{x}_{r}^{o} \times \mathbf{d}_{2 o}^{o}\right)
$$

The expected length of the drive 4 and its versor are obtained from the triangle $A_{4} A_{2} C_{4}$ :

$$
s_{4 o}=\sqrt{\left(\mathbf{s}_{4 o} \cdot \mathbf{x}_{r}^{o}\right)^{2}+\left(\mathbf{s}_{4 o} \cdot \mathbf{y}_{r}^{o}\right)^{2}+\left(\mathbf{s}_{4 o} \cdot \mathbf{z}_{r}^{o}\right)^{2}}, \quad \mathbf{s}_{4 o}^{o}=\frac{\mathbf{s}_{4 o}}{s_{4 o}}
$$

where: $\mathbf{s}_{4 o}=c_{2} \mathbf{c}_{2 o}^{o}-a_{4} \mathbf{a}_{4}^{o}$ - vector of the drive 4 .

The expected length of the actuator and its axis versor are obtained on the basis of a polygon $A_{1} A_{2} B_{2} O_{p} B_{1}$ : 


$$
s_{1 o}=\sqrt{\left(\mathbf{s}_{1 o} \cdot \mathbf{x}_{r}^{o}\right)^{2}+\left(\mathbf{s}_{1 o} \cdot \mathbf{y}_{r}^{o}\right)^{2}+\left(\mathbf{s}_{1 o} \cdot \mathbf{z}_{r}^{o}\right)^{2}}, \quad \mathbf{s}_{1 o}^{o}=\frac{\mathbf{s}_{1 o}}{s_{1 o}}
$$

where: $\mathbf{s}_{1 o}=d_{2} \mathbf{d}_{2 o}^{o}+b_{2} \mathbf{y}_{p o}^{o}-a_{1} \mathbf{a}_{1}^{o}-b_{1} \mathbf{x}_{p o}^{o}$ - vector of the actuator 1 .

\subsection{Direct kinematics problem of links velocity}

In order to solve the simple problem to derive velocity of the active suspension system in the coordinate system associated with the frame it is required that the following vectors have to be determined:

$\boldsymbol{\omega}_{p, r}=\boldsymbol{\omega}_{k, r}=\boldsymbol{\omega}_{f, r}$ - identical angular velocity of the platform $p$, of the cab $k$ and the operator seat $f$,

$\mathbf{v}_{O_{k}, r}$ - linear velocity of the cab's c.o.g $Q_{k}$,

$\mathbf{v}_{O_{f}, r}$ - linear velocity of the c.o.g. in the seat-operator system $O_{f}$ as functions of cylinders' velocity.

The kinematic chain determined by points $A_{4} C_{4} A_{2}$ (Fig 5) satisfies the closing condition:

$$
a_{4} \mathbf{a}_{4}^{o}+s_{4} \mathbf{s}_{4}^{o}=c_{2} \mathbf{c}_{2}^{o}
$$

Differentiating Eq (28) over time yields:

$$
\dot{s}_{4} \mathbf{s}_{4}^{o}+s_{4}\left(\boldsymbol{\omega}_{4, r} \times \mathbf{s}_{4}^{o}\right)=c_{2}\left(\boldsymbol{\omega}_{2, r} \times \mathbf{c}_{2}^{o}\right)
$$

The versor $\mathbf{x}_{r}^{o}$ determines the direction of the angular velocity vector of the rocker arm 2 :

$$
\omega_{2, r}=\omega_{2, r} \mathbf{x}_{r}^{o}
$$

Scalar-multiplying Eq (29) by $\mathbf{s}_{4}^{o}$ yields:

$$
\omega_{2, r}=\frac{\dot{s}_{4}}{r_{2}}
$$

where: $r_{2}=c_{2}\left[\left(\mathbf{x}_{r}^{o} \times \mathbf{c}_{2}^{o}\right) \cdot \mathbf{s}_{4}^{o}\right]$.

The kinematic chain determined by points $A_{2} B_{2} B_{3} A_{3}$ (Fig 5.) satisfies the closing condition:

$$
d_{2} \mathbf{d}_{2}^{o}+b_{23} \mathbf{y}_{p}^{o}=a_{23} \mathbf{y}_{r}^{o}+d_{3} \mathbf{d}_{3}^{o}
$$

Differentiating Eq (32) over time yields:

$$
d_{2}\left(\boldsymbol{\omega}_{2, r} \times \mathbf{d}_{2}^{o}\right)+b_{23}\left(\boldsymbol{\omega}_{p, r} \times \mathbf{y}_{p}^{o}\right)=d_{3}\left(\boldsymbol{\omega}_{3, r} \times \mathbf{d}_{3}^{o}\right)
$$

The versor $\mathbf{x}_{r}^{o}$ determines the direction of the angular velocity vector of the rocker arm 3 : 


$$
\boldsymbol{\omega}_{3, r}=\omega_{3, r} \mathbf{x}_{r}^{o}
$$

Substituting (30), (31) and (34) into Eq (33) and scalar-multiplying all vectors by the versor $\mathbf{y}_{p}^{o}$, yields the modulus of angular velocity vector $\omega_{3, r}$ :

$$
\omega_{3, r}=\frac{\dot{s}_{4}}{r_{3}}
$$

where: $r_{3}=\frac{c_{2} d_{3}}{d_{2}} \frac{\left[\left(\mathbf{x}_{r}^{o} \times \mathbf{d}_{3}^{o}\right) \cdot \mathbf{y}_{p}^{o}\right]\left[\left(\mathbf{x}_{r}^{o} \times \mathbf{c}_{2}^{o}\right) \cdot \mathbf{s}_{4}^{o}\right]}{\left(\mathbf{x}_{r}^{o} \times \mathbf{d}_{2}^{o}\right) \cdot \mathbf{y}_{p}^{o}}$.

The angular velocity vector of the platform $\mathrm{p}$ can be expressed as the sum of three components whose axis directions are determined by versors $\mathbf{x}_{p}^{o}, \mathbf{y}_{p}^{o}$ and $\mathbf{z}_{p}^{o}$ :

$$
\boldsymbol{\omega}_{p, r}=\omega_{(p, r) x_{p}} \mathbf{x}_{p}^{o}+\omega_{(p, r) y_{p}} \mathbf{y}_{p}^{o}+\omega_{(p, r) z_{p}} \mathbf{z}_{p}^{o}
$$

Recalling Eq (30), (31), (34), (35), (36), Eq (33) can be rewritten as:

$$
d_{2}\left(\frac{\dot{s}_{4}}{r_{2}} \mathbf{x}_{r}^{o} \times \mathbf{d}_{2}^{o}\right)+b_{23} \omega_{(p, r) x_{p}} \mathbf{z}_{p}^{o}-b_{23} \omega_{(p, r) z_{p}} \mathbf{x}_{p}^{o}=d_{3}\left(\frac{\dot{s}_{4}}{r_{3}} \mathbf{x}_{r}^{o} \times \mathbf{d}_{3}^{o}\right)
$$

Scalar-multiplying Eq (37) by $\mathbf{x}_{p}^{o}$ yields:

$$
\omega_{(p, r) z_{p}}=\frac{\dot{s}_{4}}{r_{p z_{p}}}
$$

where: $r_{p z_{p}}=\frac{b_{23}}{\left(\mathbf{x}_{p}^{o} \times \mathbf{x}_{r}^{o}\right) \cdot\left(\frac{d_{2}}{r_{2}} \mathbf{d}_{2}^{o}-\frac{d_{3}}{r_{3}} \mathbf{d}_{3}^{o}\right)}$.

Scalar-multiplying Eq (37) by $\mathbf{z}_{p}^{o}$ yields:

$$
\omega_{(p, r) x_{p}}=\frac{\dot{s}_{4}}{r_{p x_{p}}}
$$

where: $r_{p x_{p}}=\frac{b_{23}}{\left(\mathbf{z}_{p}^{o} \times \mathbf{x}_{r}^{o}\right) \cdot\left(\frac{d_{3}}{r_{3}} \mathbf{d}_{3}^{o}-\frac{d_{2}}{r_{2}} \mathbf{d}_{2}^{o}\right)}$.

The closed kinematic chain comprising a actuator $s_{1}$ and represented as a pentagon $A_{2} A_{1} B_{1} O_{p} B_{2}$ in Fig 5 satisfies the closing condition:

$$
a_{1} \mathbf{a}_{1}^{o}+s_{1} \mathbf{s}_{1}^{o}+b_{1} \mathbf{x}_{p}^{o}=d_{2} \mathbf{d}_{2}^{o}+b_{2} \mathbf{y}_{p}^{o}
$$


Differentiating Eq (40) over time yields:

$$
\dot{s}_{1} \mathbf{s}_{1}^{o}+s_{1}\left(\boldsymbol{\omega}_{1, r} \times \mathbf{s}_{1}^{o}\right)+b_{1}\left(\boldsymbol{\omega}_{p, r} \times \mathbf{x}_{p}^{o}\right)=d_{2}\left(\boldsymbol{\omega}_{2, r} \times \mathbf{d}_{2}^{o}\right)+b_{2}\left(\boldsymbol{\omega}_{p, r} \times \mathbf{y}_{p}^{o}\right)
$$

Recalling the relationships expressing the angular velocity vector of the platform (Eq 36) and angular velocity vector of the rocker arm 2 (Eq 30), Eq (41) can be rearranged accordingly:

$$
\dot{s}_{1} \mathbf{s}_{1}^{o}+s_{1}\left(\omega_{1, r} \times \mathbf{s}_{1}^{o}\right)+b_{1}\left(\omega_{(p, r) z_{p}} \mathbf{y}_{p}^{o}-\omega_{(p, r) y_{p}} \mathbf{z}_{p}^{o}\right)=d_{2}\left(\frac{\dot{s}_{4}}{r_{2}} \mathbf{x}_{r}^{o} \times \mathbf{d}_{2}^{o}\right)+b_{2}\left(\omega_{(p, r) x_{p}} \mathbf{z}_{p}^{o}-\omega_{(p, r) z_{p}} \mathbf{x}_{p}^{o}\right)
$$

Scalar multiplying vectors present in Eq (42) by $\mathbf{s}_{1}^{o}$ yields the coordinate of the angular velocity of the platform in the direction determined by the versor $\mathbf{y}_{p}^{o}$ :

$$
\omega_{(p, r) y_{p}}=\frac{\dot{s}_{1}}{r_{p y_{p} 1}}+\frac{\dot{s}_{4}}{r_{p y_{p} 4}}
$$

where: $r_{p y_{p} 1}=b_{1}\left(\mathbf{z}_{p}^{o} \cdot \mathbf{s}_{1}^{o}\right), r_{p y_{p} 4}=\frac{b_{1} \mathbf{z}_{p}^{o} \cdot \mathbf{s}_{1}^{o}}{\left[\frac{b_{1}}{r_{p z_{p}}} \mathbf{y}_{p}^{o}-\frac{d_{2}}{r_{2}}\left(\mathbf{x}_{r}^{o} \times \mathbf{d}_{2}^{o}\right)-\frac{b_{2}}{r_{p x_{p}}} \mathbf{z}_{p}^{o}+\frac{b_{2}}{r_{p z_{p}}} \mathbf{x}_{p}^{o}\right] \cdot \mathbf{s}_{1}^{o}}$.

The angular velocity vector of the platform, based on (36), (39), (43), (38), will become:

$$
\boldsymbol{\omega}_{p, r}=\frac{\dot{s}_{4}}{r_{p x_{p}}} \mathbf{x}_{p}^{o}+\left(\frac{\dot{s}_{1}}{r_{p y_{p} 1}}+\frac{\dot{s}_{4}}{r_{p y_{p} 4}}\right) \mathbf{y}_{p}^{o}+\frac{\dot{s}_{4}}{r_{p z_{p}}} \mathbf{z}_{p}^{o}
$$

Finally, the angular velocity vector of the platform, cab and the seat is linearly related to the velocity of actuators $s_{1}$ and $s_{4}$ :

$$
\omega_{p, r}=\omega_{k, r}=\omega_{f, r}=\dot{s}_{1} \mathbf{h}_{1}+\dot{s}_{4} \mathbf{h}_{4}
$$

where: $\mathbf{h}_{1}=\frac{\mathbf{y}_{p}^{o}}{r_{p y_{p} 1}}, \mathbf{h}_{4}=\frac{\mathbf{x}_{p}^{o}}{r_{p x_{p}}}+\frac{\mathbf{y}_{p}^{o}}{r_{p y_{p} 4}}+\frac{\mathbf{z}_{p}^{o}}{r_{p \mathbf{z}_{p}}}$.

Eq (45) can be written in the matrix format:

$$
\boldsymbol{\omega}_{p, r}=\mathbf{J}_{\omega} \dot{\mathbf{s}}
$$

where: $\mathbf{J}_{\omega}=\left[\begin{array}{lll}\mathbf{h}_{1} & \mathbf{h}_{4} & \mathbf{0}\end{array}\right], \dot{\mathbf{s}}=\left[\begin{array}{lll}\dot{s}_{1} & \dot{s}_{4} & \dot{s}_{5}\end{array}\right]^{T}$.

The radius vector of the point $O_{k}$ - the origin of the reference system associated with the frame (Fig 5), is expressed as:

$$
\mathbf{r}_{r k}=d_{2} \mathbf{d}_{2}^{o}+b_{2} \mathbf{y}_{p}^{o}+\mathbf{r}_{p k}
$$


where: $\mathbf{r}_{p k}=\mathbf{x}_{p}^{o p} r_{(p k) x}+\mathbf{y}_{p}^{o p} r_{(p k) y}+\mathbf{z}_{p}^{o p} r_{(p k) z}$.

Differentiating Eq (47) over time yields the linear velocity vector of the point $O_{k}$ :

$$
\mathbf{v}_{O_{k}, r}=d_{2}\left(\boldsymbol{\omega}_{2, r} \times \mathbf{d}_{2}^{o}\right)+\boldsymbol{\omega}_{p, r} \times\left(\mathbf{y}_{p}^{o} b_{2}+\mathbf{r}_{p k}\right)
$$

Recalling Eq (30), (31), (45), Eq (48) can be rewritten as:

$$
\mathbf{v}_{O_{k}, r}=\dot{s}_{1} \mathbf{k}_{1 O_{k}}+\dot{s}_{4} \mathbf{k}_{4 O_{k}}
$$

where: $\mathbf{k}_{1 O_{k}}=\mathbf{h}_{1} \times\left(b_{2} \mathbf{y}_{p}^{o}+\mathbf{r}_{p k}\right), \mathbf{k}_{4 O_{k}}=\frac{d_{2}}{r_{2}}\left(\mathbf{x}_{r}^{o} \times \mathbf{d}_{2}^{o}\right)+\mathbf{h}_{4} \times\left(b_{2} \mathbf{y}_{p}^{o}+\mathbf{r}_{p k}\right)$.

Eq (49) can be expressed in the matrix format:

$$
\mathbf{v}_{O_{k}, r}=\mathbf{J}_{v k} \dot{\mathbf{s}}
$$

where: $\mathbf{J}_{v k}=\left[\begin{array}{lll}\mathbf{k}_{1 O_{k}} & \mathbf{k}_{4 O_{k}} & \mathbf{0}\end{array}\right]$.

The radius vector of the c.o.g of the seat-operator system $O_{f}$ becomes:

$$
\mathbf{r}_{r f}=d_{2} \mathbf{d}_{2}^{o}+b_{2} \mathbf{y}_{p}^{o}+\mathbf{r}_{p f}
$$

where: $\mathbf{r}_{p f}=\mathbf{x}_{p}^{o p} r_{(p f) x}+\mathbf{y}_{p}^{o p} r_{(p f) y}+\mathbf{z}_{p}^{o}\left(s_{5}+d f_{z}\right)$.

Differentiating Eq (51) over time yields the linear velocity of the point $O_{f}$.

$$
\mathbf{v}_{O_{f}, r}=d_{2} \boldsymbol{\omega}_{2, r} \times \mathbf{d}_{2}^{o}+\boldsymbol{\omega}_{p, r} \times\left(\mathbf{y}_{p}^{o} b_{2}+\mathbf{r}_{p f}\right)+\dot{s}_{5} \mathbf{z}_{p}^{o}
$$

Recalling Eq (30), (31), (45), Eq (52) can be rewritten as:

$$
\mathbf{v}_{O_{f}, r}=\dot{s}_{1} \mathbf{k}_{1 O_{f}}+\dot{s}_{4} \mathbf{k}_{4 O_{f}}+\dot{s}_{5} \mathbf{k}_{5 O_{f}}
$$

where: : $\mathbf{k}_{1 O_{f}}=\mathbf{h}_{1} \times\left(b_{2} \mathbf{y}_{p}^{o}+\mathbf{r}_{p f}\right), \mathbf{k}_{4 O_{f}}=\frac{d_{2}}{r_{2}}\left(\mathbf{x}_{r}^{o} \times \mathbf{d}_{2}^{o}\right)+\mathbf{h}_{4} \times\left(\mathbf{y}_{p}^{o} b_{2}+\mathbf{r}_{p f}\right), \mathbf{k}_{5 O_{f}}=\mathbf{z}_{p}^{o}$.

Eq (53) can be expressed in the matrix format:

$$
\mathbf{v}_{O_{f}, r}=\mathbf{J}_{v f} \dot{\mathbf{s}}
$$

where: $\mathbf{J}_{v f}=\left[\begin{array}{lll}\mathbf{k}_{1 O_{f}} & \mathbf{k}_{4 O_{f}} & \mathbf{k}_{5 O_{f}}\end{array}\right]$.

To define the operating conditions of the drives in the active suspension mechanism, velocity vectors related to the road system are of key importance. The absolute angular velocity of the cab in the reference system associated with the frame becomes:

$$
\boldsymbol{\omega}_{p, g}=\boldsymbol{\omega}_{p, r}+\boldsymbol{\omega}_{r, g}=\mathbf{J}_{\omega} \dot{\mathbf{s}}+\boldsymbol{\omega}_{r, g}
$$


where: $\omega_{r, g}$ - angular velocity of the frame with respect to road, based on measurement data. Absolute linear velocities of points $O_{k}$ and $O_{f}$ expressed in the reference system associated with the frame are:

$$
\begin{aligned}
& \mathbf{v}_{O_{k}, g}=\mathbf{v}_{O_{m}, g}+\boldsymbol{\omega}_{r, g} \times\left(\mathbf{r}_{m r}+\mathbf{r}_{r k}\right)+\mathbf{v}_{O_{k}, r} \\
& \mathbf{v}_{O_{f}, g}=\mathbf{v}_{O_{m}, g}+\boldsymbol{\omega}_{r, g} \times\left(\mathbf{r}_{m r}+\mathbf{r}_{r f}\right)+\mathbf{v}_{O_{f}, r}
\end{aligned}
$$

where: $\mathbf{v}_{O_{m}, g}$ - measured linear velocity of the control point $O_{m}$ associated with the frame with respect to the road; $\mathbf{r}_{m r}=\left[r_{(m r) x}, r_{(m r) y}, r_{(m r) z}\right]^{T}$ - vector between the points $O_{m}$ and $O r$ expressed in the reference system associated with the frame.

\subsection{Inverse kinematics problem of links velocity}

The inverse problem involves finding the drive velocities for the predetermined cab velocity with respect to the road. As the active suspension mechanism displays three degrees of freedom (DOFs), three constraints can be imposed upon the cab velocity. The function of the active suspension system is to stabilise the cab in the vertical direction, hence the condition is adopted prohibiting the absolute rotating motion of the platform around its two axes $\mathbf{x}_{p o}^{o}$ and $\mathbf{y}_{p o}^{o}$. The third condition implicates that the absolute value of linear velocity of the point $O_{f}$ in the direction of the gravity force should be zero:

$$
\begin{aligned}
& \boldsymbol{\omega}_{(p, g) o} \cdot \mathbf{x}_{p o}^{o}=0 \\
& \boldsymbol{\omega}_{(p, g) o} \cdot \mathbf{y}_{p o}^{o}=0 \\
& \mathbf{v}_{\left(O_{f}, g\right) o} \cdot \mathbf{z}_{g}^{o}=0
\end{aligned}
$$

Recalling Eq (55) and conditions (58), (59), we get the formulas expressing the expected velocities of actuators 1 and 4 :

$$
\left.\begin{array}{l}
\dot{s}_{1 o} \mathbf{h}_{1 o} \cdot \mathbf{x}_{p o}^{o}+\dot{s}_{4 o} \mathbf{h}_{4 o} \cdot \mathbf{x}_{p o}^{o}+\boldsymbol{\omega}_{r, g} \cdot \mathbf{x}_{p o}^{o}=0 \\
\dot{s}_{10} \mathbf{h}_{1 o} \cdot \mathbf{y}_{p o}^{o}+\dot{s}_{4 o} \mathbf{h}_{4 o} \cdot \mathbf{y}_{p o}^{o}+\boldsymbol{\omega}_{r, g} \cdot \mathbf{y}_{p o}^{o}=0
\end{array}\right\}
$$

When the actuators 1 and 4 should move at velocities governed by Eq (61), the cab will perform a slight rotating motion around in the direction $\mathbf{z}_{p o}^{o}$ only.

The third condition (60) in relation to $\mathrm{Eq}(54)$, (57) gives:

$$
\dot{s}_{1 o}\left(\mathbf{k}_{1 O_{f}} \cdot \mathbf{z}_{g}^{o}\right)+\dot{s}_{4 o}\left(\mathbf{k}_{4 O_{f}} \cdot \mathbf{z}_{g}^{o}\right)+\dot{s}_{5 o}\left(\mathbf{k}_{5 O_{f}} \cdot \mathbf{z}_{g}^{o}\right)+\mathbf{v}_{O_{m}, g} \cdot \mathbf{z}_{g}^{o}+\left[\boldsymbol{\omega}_{r, g} \times\left(\mathbf{r}_{m r}+\mathbf{r}_{(r f) o}\right)\right] \cdot \mathbf{z}_{g}^{o}=0
$$

The solution to linear system of equations (61) and (62) can be written as a matrix equation:

$$
\dot{\mathbf{s}}_{o}=\mathbf{J v}
$$


where: $\dot{\mathbf{s}}_{o}=\left[\begin{array}{c}\dot{s}_{1 o} \\ \dot{s}_{4 o} \\ \dot{s}_{5 o}\end{array}\right], \mathbf{J}=-\left[\begin{array}{ccc}\mathbf{h}_{1 o} \cdot \mathbf{x}_{p o}^{o} & \mathbf{h}_{4 o} \cdot \mathbf{x}_{p o}^{o} & 0 \\ \mathbf{h}_{10} \cdot \mathbf{y}_{p o}^{o} & \mathbf{h}_{4 o} \cdot \mathbf{y}_{p o}^{o} & 0 \\ \mathbf{k}_{1 O_{f}} \cdot \mathbf{z}_{g}^{o} & \mathbf{k}_{4 O_{f}} \cdot \mathbf{z}_{g}^{o} & \mathbf{k}_{5 O_{f}} \cdot \mathbf{z}_{g}^{o}\end{array}\right]^{-1}\left[\begin{array}{cc}\mathbf{0}_{1 \times 3} & \mathbf{x}_{p o}^{o} \\ \mathbf{0}_{1 \times 3} & \mathbf{y}_{p o}^{o} \\ \mathbf{z}_{g}^{o} & \left(\mathbf{r}_{m r}+\mathbf{r}_{r f}\right) \times \mathbf{z}_{g}^{o}\end{array}\right], \mathbf{v}=\left[\begin{array}{c}\mathbf{v}_{O_{m}, g} \\ \boldsymbol{\omega}_{r, g}\end{array}\right]$.

\subsection{Constraining the motion of the drive responsible for the seat movement in the vertical direction}

The machine, when in service or during the ride, may change its position in the vertical direction such that in order to stabilise the seat position in this direction the operating range of the actuator 5 should be exceeded. To solve this problem, it is suggested that a penalty function should be introduced, its argument being the instantaneous length of the actuator s5:

$$
\dot{s}_{5 k}=K_{5} \dot{s}_{5 k \max }\left[\frac{s_{5 \dot{s} r}-s_{5}}{0.5\left(s_{5 \max }-s_{5 \min }\right)}\right]^{2 n-1}
$$

where: $s_{5 \dot{s} r}=0.5\left(s_{5 \max }+s_{5 \min }\right), K_{5}$ - amplification factor penalty function $\dot{s}_{5 k \max }$ - maximal velocity of the actuator, $n \in N$.

The final expected velocity of the actuator 5 should involve a term responsible for the seat "drifting" towards the middle position:

$$
\dot{s}_{5 o}^{*}=\dot{s}_{5 o}+\dot{s}_{5 k}
$$

where: $\dot{s}_{50}$ - expected velocity in the actuator 5, derived from formula (63).

The second term in (65) represents the seat movement towards the middle position being superimposed on its relative movement. The assumed penalty function (64) guarantees that the relative velocity during the seat's return movement (Fig 7) to the middle position should be significant at extreme points of the actuator's displacement range.

\subsection{Cab and seat acceleration}

In order to solve the simple problem involving acceleration of the active suspension mechanism in the reference system associated with the frame, it is required that certain quantities should be determined in the function of length, velocity and acceleration of actuators 1,4,5. These include: $\varepsilon_{p, r}$ - angular acceleration of the platform, cab and seat, $\mathbf{a}_{O_{k}, r}$ linear acceleration of the cab's c.o.g, $\mathbf{a}_{O_{f}, r}$ - linear acceleration of the c.o.g of the seatoperator system. Differentiating Eq (46), (50), (54) with respect to time yields the angular acceleration of the platform, cab and seat and linear acceleration at points $O_{k}$ and $O_{f}$.

$$
\begin{gathered}
\varepsilon_{p, r}=\dot{\mathbf{J}}_{\omega} \dot{\mathbf{s}}+\mathbf{J}_{\omega} \ddot{\mathbf{s}} \\
\mathbf{a}_{O_{k}, r}=\dot{\mathbf{J}}_{v k} \dot{\mathbf{s}}+\mathbf{J}_{v k} \ddot{\mathbf{s}}
\end{gathered}
$$




$$
\mathbf{a}_{O_{f}, r}=\dot{\mathbf{J}}_{v f} \dot{\mathbf{s}}+\mathbf{J}_{v f} \ddot{\mathbf{s}}
$$

where: $\ddot{\mathbf{s}}=\left[\begin{array}{lll}\ddot{\mathbf{s}}_{1} & \ddot{\mathbf{s}}_{4} & \ddot{\mathbf{s}}_{5}\end{array}\right]^{T}$.

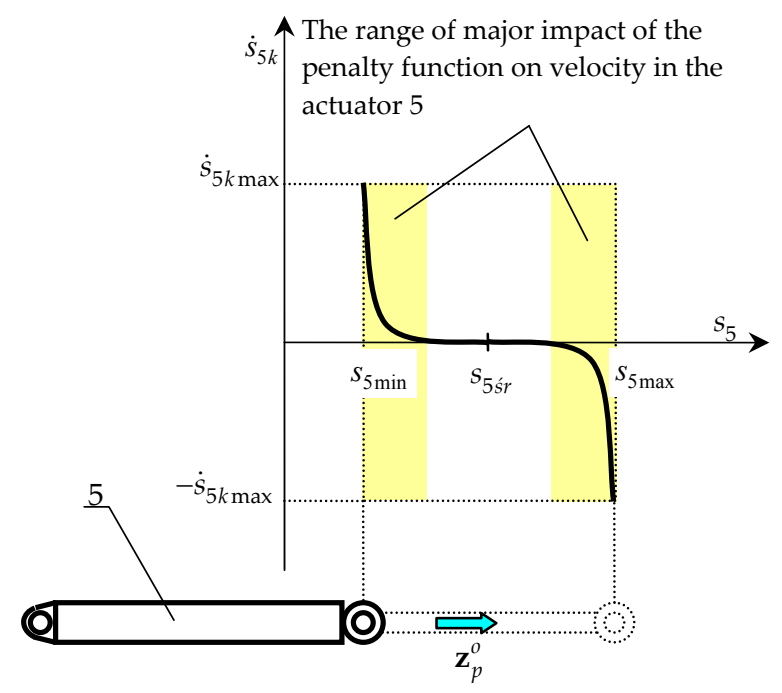

Figure 7. Penalty function

The inertia loads are determined basing on absolute acceleration values related to the inertial reference system $\left\{O_{8} x_{8} y_{8} z_{8}\right\}$. In accordance with Eq (55), the absolute angular accelerations of the platform, cab and seat expressed in the mobile reference system associated with the frame become:

$$
\varepsilon_{p, g}=\varepsilon_{k, g}=\varepsilon_{f, g}=\varepsilon_{r, g}+\varepsilon_{p, r}+\omega_{r, g} \times \omega_{p, r}
$$

Recalling Eq (56), (57), the absolute linear acceleration of the points $O_{r}, O_{k}$ and $O_{f}$ in the mobile reference system associated with the frame become:

$$
\begin{gathered}
\mathbf{a}_{O_{r}, g}=\mathbf{a}_{O_{m}, g}+\varepsilon_{r, g} \times \mathbf{r}_{m r}+\omega_{r, g} \times\left(\omega_{r, g} \times \mathbf{r}_{m r}\right) \\
\mathbf{a}_{O_{k}, g}=\mathbf{a}_{O_{r}, g}+\varepsilon_{r, g} \times \mathbf{r}_{r k}+2 \boldsymbol{\omega}_{r, g} \times \mathbf{v}_{O_{k}, r}+\boldsymbol{\omega}_{r, g} \times\left(\boldsymbol{\omega}_{r, g} \times \mathbf{r}_{r k}\right)+\mathbf{a}_{O_{k}, r} \\
\mathbf{a}_{O_{f}, g}=\mathbf{a}_{O_{k}, g}+\boldsymbol{\varepsilon}_{k, g} \times \mathbf{r}_{k f}+2 \boldsymbol{\omega}_{k, g} \times \mathbf{v}_{O_{f}, k}+\boldsymbol{\omega}_{k, g} \times\left(\boldsymbol{\omega}_{k, g} \times \mathbf{r}_{k f}\right)+\mathbf{a}_{O_{f}, k}
\end{gathered}
$$

where: $\mathbf{a}_{O_{m}, g}$ - measured linear acceleration of a point on the frame $Q_{m}$ with respect to the inertial reference system $\left\{O_{g} x_{8} y_{g} z_{g}\right\}, \omega_{r, g}, \varepsilon_{r, g}$ - measured angular velocity and acceleration of the machine frame with respect to the inertial reference system $\left\{O_{g} x_{8} y_{g} z_{g}\right\}$, $\boldsymbol{\omega}_{k, g}=\boldsymbol{\omega}_{p, g}=\boldsymbol{\omega}_{r, g}+\boldsymbol{\omega}_{p, r}, \mathbf{r}_{k f}=\overrightarrow{O_{k} O_{f}}, \mathbf{r}_{r k}=\overrightarrow{O_{r} O_{k}}, \mathbf{r}_{m r}=\overrightarrow{O_{m} O_{r}}$. 


\section{Inverse problem of dynamics}

The external loads acting on the active suspension mechanism involve the gravity forces, inertia forces and moments of inertial force of the platform together with the cab, the seat and the operator. These are governed by the Newton-Euler equations, referenced in [18]:

$$
\begin{gathered}
\mathbf{P}_{b k}=-m_{k} \mathbf{a}_{O_{k}, g} \\
\mathbf{M}_{b k}=-\boldsymbol{\varepsilon}_{k, g} \mathbf{I}_{k}-\tilde{\boldsymbol{\omega}}_{k, g} \mathbf{I}_{k} \boldsymbol{\omega}_{k, g} \\
\mathbf{P}_{b f}=-m_{f} \mathbf{a}_{O_{f}, g} \\
\mathbf{M}_{b f}=-\boldsymbol{\varepsilon}_{f, g} \mathbf{I}_{f}-\tilde{\boldsymbol{\omega}}_{f, g} \mathbf{I}_{f} \boldsymbol{\omega}_{f, g}
\end{gathered}
$$

where: $\tilde{\boldsymbol{\omega}}=\left[\begin{array}{ccc}0 & -\omega_{z} & \omega_{y} \\ \omega_{z} & 0 & -\omega_{x} \\ -\omega_{y} & \omega_{x} & 0\end{array}\right], \mathbf{I}_{k}={ }_{p}^{r} \mathbf{R}{ }^{k} \mathbf{I}_{k}{ }_{p}^{r} \mathbf{R}^{T}, \mathbf{I}_{f}={ }_{p}^{r} \mathbf{R}{ }^{f} \mathbf{I}_{f}{ }_{p}^{r} \mathbf{R}^{T}$,

${ }^{k} \mathbf{I}_{k},{ }^{f} \mathbf{I}_{f}$ - mass moments of inertia of the cab and the seat with operator in their own reference systems.

The sum total of instantaneous power applied by the active suspension mechanism and power of the gravity and inertia forces are brought down to zero:

$$
\begin{gathered}
\dot{\mathbf{s}}^{T} \mathbf{F}_{s}+m_{k} \mathbf{v}_{O_{k}, r}^{T}\left(\mathbf{g}-\mathbf{a}_{O_{k}, g}\right)+\boldsymbol{\omega}_{k, r}^{T}\left(-\mathbf{I}_{k} \boldsymbol{\varepsilon}_{k, g}-\tilde{\boldsymbol{\omega}}_{k, g} \mathbf{I}_{k} \boldsymbol{\omega}_{k, g}\right)+ \\
+m_{f} \mathbf{v}_{O_{f}, r}^{T}\left(\mathbf{g}-\mathbf{a}_{O_{f}, g}\right)+\boldsymbol{\omega}_{f, r}^{T}\left(-\mathbf{I}_{f} \boldsymbol{\varepsilon}_{f, g}-\tilde{\boldsymbol{\omega}}_{f, g} \mathbf{I}_{f} \boldsymbol{\omega}_{f, g}\right)=0
\end{gathered}
$$

where: $\mathbf{F}_{s}=\left[F_{1}, F_{4}, F_{5}\right]^{T}$ - forces developed by the drives.

Recalling Jacobean matrices (46), (50), (54), Eq (77) can be rewritten as:

$$
\begin{gathered}
\dot{\mathbf{s}}^{T}\left[\mathbf{F}_{s}+m_{k} \mathbf{J}_{v k}^{T}\left(\mathbf{g}-\mathbf{a}_{O_{k}, g}\right)+\mathbf{J}_{\omega}^{T}\left(-\mathbf{I}_{k} \boldsymbol{\varepsilon}_{k, g}-\tilde{\boldsymbol{\omega}}_{k, g} \mathbf{I}_{k} \boldsymbol{\omega}_{k, g}\right)+\right. \\
\left.+m_{f} \mathbf{J}_{v f}^{T}\left(\mathbf{g}-\mathbf{a}_{O_{f}, g}\right)+\mathbf{J}_{\omega}^{T}\left(-\mathbf{I}_{f} \boldsymbol{\varepsilon}_{f, g}-\tilde{\boldsymbol{\omega}}_{f, g} \mathbf{I}_{f} \boldsymbol{\omega}_{f, g}\right)\right]=0
\end{gathered}
$$

Knowing the loads due to gravity and inertia, Eq (78) yields the forces acting in the drives:

$$
\mathbf{F}_{s}=m_{k} \mathbf{J}_{v k}^{T}\left(\mathbf{a}_{O_{k}, g}-\mathbf{g}\right)+m_{f} \mathbf{J}_{v f}^{T}\left(\mathbf{a}_{O_{f}, g}-\mathbf{g}\right)+\mathbf{J}_{\omega}^{T}\left\{\left(\mathbf{I}_{k}+\mathbf{I}_{f}\right) \varepsilon_{k, g}+\left[\tilde{\boldsymbol{\omega}}_{k, g}\left(\mathbf{I}_{f}+\mathbf{I}_{k}\right) \boldsymbol{\omega}_{k, g}\right]\right\}
$$

\section{Simulation of the active suspension system}

The operation of the active suspension system is investigated using two mutually supportive programmes. MSC visualNastran $4 \mathrm{D}$ is used to develop the model of the input 
inducing the machine motion, of the machine suspension, the active suspension mechanism for the cab and the seat. All these modelled elements are simplified (Fig 8).

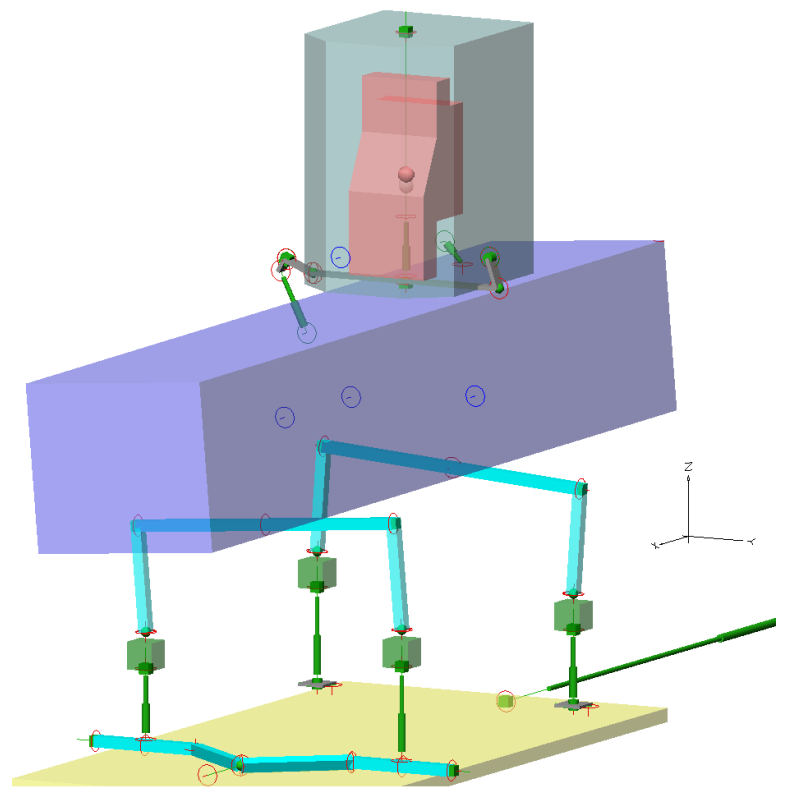

Figure 8. Model of the road input, machine suspension mechanism, active suspension of the cab and seat developed in MSC visualNASTRAN 4D

The programme enables the measurements of the actuator length, the angle of frame tilting $\alpha_{x}$ and pitching $\alpha_{y}$, velocity and acceleration $O_{m}$ and of velocity and acceleration of the $\mathrm{cab}^{\prime}$ s angular motion. These are shown in the block diagram "Measurements of the machine frame movements". During the simulation procedure, these quantities are sent to be further handled by Matlab/Simulink (Fig 9).

The proposed control strategy to be applied to the active suspension of the cab uses the feedback control system with compensation for the measured disturbances in the form of the machine frame movements. The expected states of the cab motion, determined in the block "Preset cab motion" involve the requirement whereby the cab is to be stabilised in the vertical direction and the seat must not be displaced along the cab's vertical axis, at the same time the operating range of the actuator 5 should be duly taken into account. Once frame movements are known from measurements and assumptions as to the anticipated cab movements being taken into account, an unambiguous procedure is applied to compute drive movements in the active suspension mechanism. On the output from the block "Inverse problem of kinematics of the active suspension mechanism" we get the expected velocities and accelerations of three drives, represented by vectors $\mathbf{s}_{o}=\left[\begin{array}{lll}s_{10} & s_{4 o} & s_{50}\end{array}\right]^{T}, \dot{\mathbf{s}}_{o}=\left[\begin{array}{lll}\dot{s}_{10} & \dot{s}_{40} & \dot{s}_{50}\end{array}\right]^{T}$. Actuators should be equipped with sensors for measuring their actual lengths $\mathbf{s}=\left[\begin{array}{lll}s_{1} & s_{4} & s_{5}\end{array}\right]^{T}$ in order to determine the control error 
$\mathbf{e}=\left[\begin{array}{lll}s_{10}-s_{1} & s_{4 o}-s_{4} & s_{5 o}-s_{5}\end{array}\right]^{T}$. The control error should tend to zero if the velocities implemented in actuators are in accordance with the formula:

$$
\dot{\mathbf{s}}_{w}=\dot{\mathbf{s}}_{o}+\mathbf{K}_{P} \mathbf{e}
$$

where: $\mathbf{K}_{P}=\left[\begin{array}{ccc}k_{p 1} & 0 & 0 \\ 0 & k_{p 4} & 0 \\ 0 & 0 & k_{p 5}\end{array}\right]$ - gain matrix in the position path.

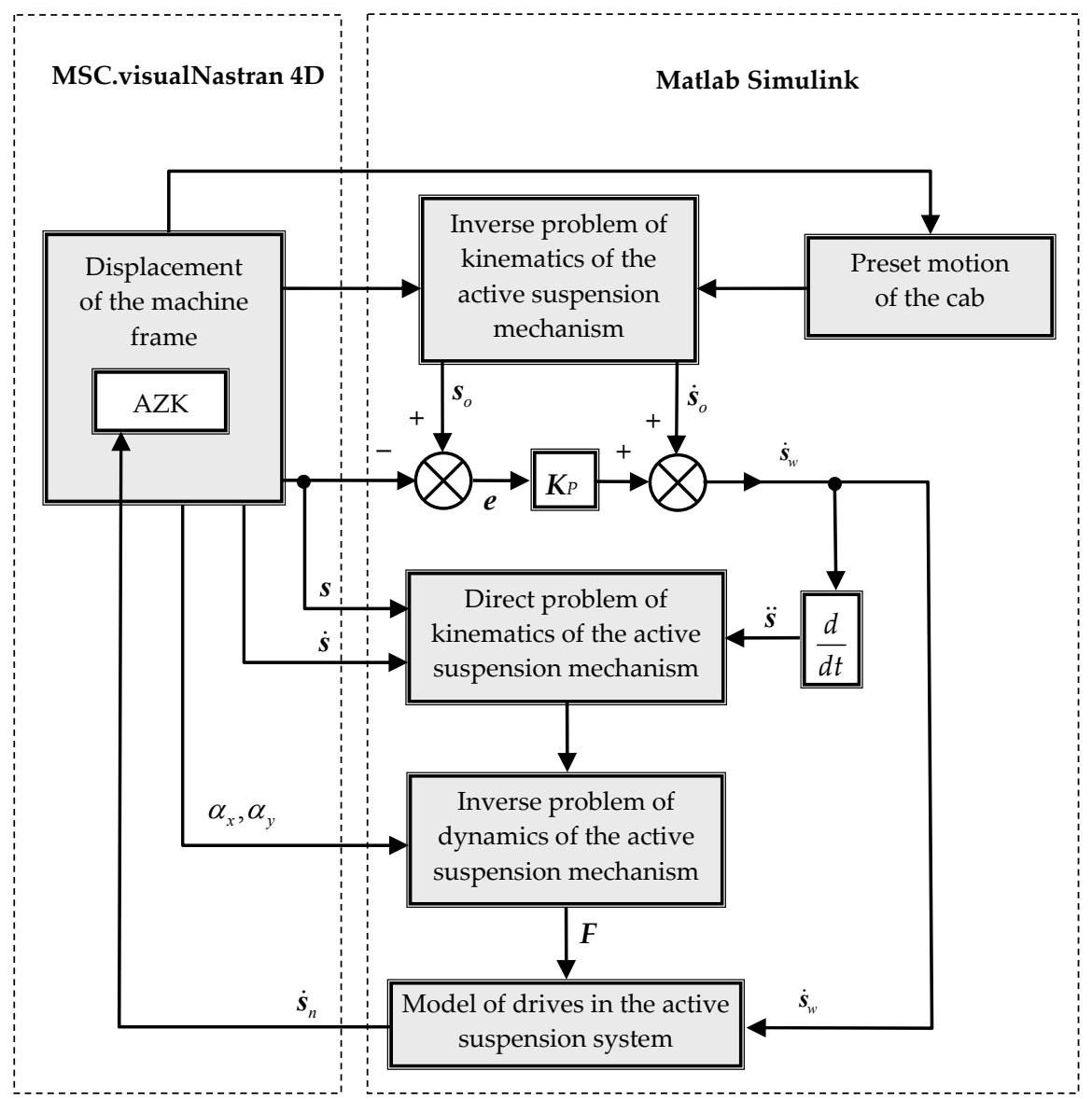

Figure 9. Model of drives control in the active suspension system - schematic diagram

Computed accelerations $\ddot{\mathbf{s}}$ and measured velocities $\dot{\mathbf{s}}$ and displacements $\mathbf{s}$ of the drives become the inputs to the block "Direct problem of kinematics of the active suspension mechanism problem of kinematics of the active suspension", which calculates the 
anticipated movement of the active suspension mechanism of the cab and of the cab itself. Basing on anticipated cab movements, inertia interactions are found which, alongside the gravity forces, become the major loads applied to the cab. The inverse problem of the active suspension dynamics involves the calculation of the driving forces in the form of a vector $\mathbf{F}=\left[\begin{array}{llll}F_{1} & F_{4} & F_{5}\end{array}\right]^{T}$, counterbalancing the external loads. The contribution of gravity forces to the load of particular drives depends on the frame tilt angles: $\alpha_{x}, \alpha_{y}$. Basing on computed loads $\boldsymbol{F}$ and the required instantaneous velocities $\dot{\mathbf{s}}_{w}$, the block "Model of active suspension drives" generates the realisable instantaneous velocities of actuators $\dot{\mathbf{s}}_{n}=\left[\begin{array}{lll}\dot{s}_{n 1} & \dot{s}_{n 4} & \dot{s}_{n 5}\end{array}\right]^{T}$. Velocity values $\dot{\mathbf{s}}_{n}$ are sent to be further processed by MSCvisualNASTRAN 4D. This work does not include the analysis of the drive model. It is assumed in simulations $\dot{\mathbf{s}}_{n}=\dot{\mathbf{s}}_{w}$.

The control of the active suspension system gives rise to certain errors $\boldsymbol{e}$, and in consequence the constraints imposed on the angular velocity of the cab and linear velocity of the cab and the seat cannot be accurately reproduced. These errors are attributable to inaccurate measurements of the frame movements, the time delay involved in implementation of the drive velocity or the drives' failure to implement the required velocity (moving beyond the limits of their typical operating range).

\section{Dependings of link dimensions of the active suspension cab mechanism}

It is demonstrated in [19] that dimensions of key parts of the mechanism $A_{2} A_{3} B_{3} B_{2}$ can be chosen such that the instantaneous centre of the platform rotation with respect to the machine frame $C_{p r}$ should be included in the road unevenness path when the active suspension system is on. When this condition is satisfied, the actuator 4, controlled in accordance with the cab vertical stabilisation requirement, will at the same time reduce the absolute movement of the platform in the direction transverse to the ride.

Assuming the central position of the cab on the machine frame, the points $A_{2}$ and $A_{3}$ should be arranged symmetrically with respect to the frame's longitudinal axis and the lengths of the rocker arms 2 and 3 should be identical $d_{2}=d_{3}=d$.

The road unevenness range and a typical crescent - shaped field of instantaneous centres of the platform rotation with respect to the frame $C_{p r}$ are shown in Fig 10. It is assumed that when the machine frame is in a horizontal position, $C_{p r}$ is found on the line of wheelground contact. The distance of joints in the rocker arm connections $A_{2}$ and $A_{3}$ from the ground is $h_{m}$. The relevant dimensions are related as follows:

$$
h^{2}+\left(\frac{a_{3}-b_{23}}{2}\right)^{2}=d^{2}, \quad \frac{h_{m}}{a_{3}}=\frac{h_{m}-h}{b_{23}}
$$

where: $h$ - distance between the platform and the points of joints $A_{2}$ and $A_{3}$ for the frame in the horizontal position. 


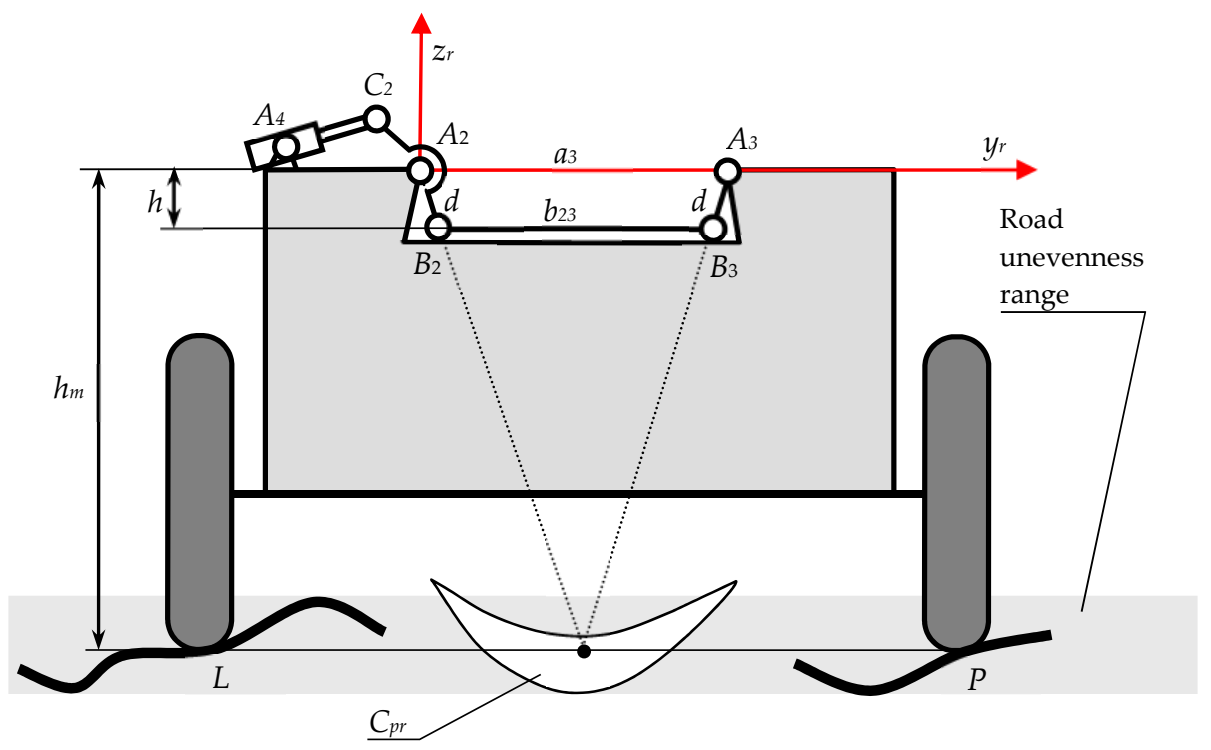

Figure 10. Distribution of the field made of points $C_{p r}$

Eliminating $h$ from Eq (81) yields the relationship between the dimension of the four bar linkage $A_{2} A_{3} B_{3} B_{2}$ and $h_{m}$. When this condition is satisfied, $C_{p r}$ is found in the road unevenness path:

$$
h_{m}\left(1-\frac{b_{23}}{a_{3}}\right)=\sqrt{d^{2}-\left(\frac{a_{3}-b_{23}}{2}\right)^{2}}
$$

Displacements of the four bar linkage in the active suspension mechanism are constrained by the occurrence of singular positions. The mechanism should not come near the singular position, when controllability of the system deteriorates and the loads acting upon the drives and mobile connections tend to increase. For the predetermined maximal height of the road unevenness range $2 h_{g}$ and for the machine wheel spacing $w_{m}$, the maximal angle $\alpha_{\max }$ a of the machine tilting with respect to the axis $y_{8}$ should be such that the four bar linkage should not assume a singular position (Fig 9):

$$
\alpha_{x \max }<\varphi \rightarrow \arcsin \frac{2 h_{g}}{w_{m}}<\arccos \frac{a_{3}^{2}+\left(b_{23}+d\right)^{2}-d^{2}}{2 a_{3}\left(b_{23}+d\right)}
$$

When the dimensions of links in the four bar linkage $A_{2} A_{3} B_{3} B_{2}$ as well as $h_{g}$ satisfy the condition (83), the mechanism is able to operate in a single configuration.

Another geometric condition stems from the assumption that the cab can move freely without colliding with the joints $A_{2}, A_{3}$, when the machine assumes its extreme position due to tilting by the angle $\alpha_{x \max }$ (Fig 9): 


$$
a_{3} \cos \alpha_{x \max }>\frac{b_{23}}{2}+\frac{w_{k}}{2}+\delta_{w}+d
$$

where: $w_{k}$ - cab width, $w_{k}<b_{23}, \delta_{w}$ - allowable distance between cab walls and the point of the joint $A_{2}$ or $A_{3}$.

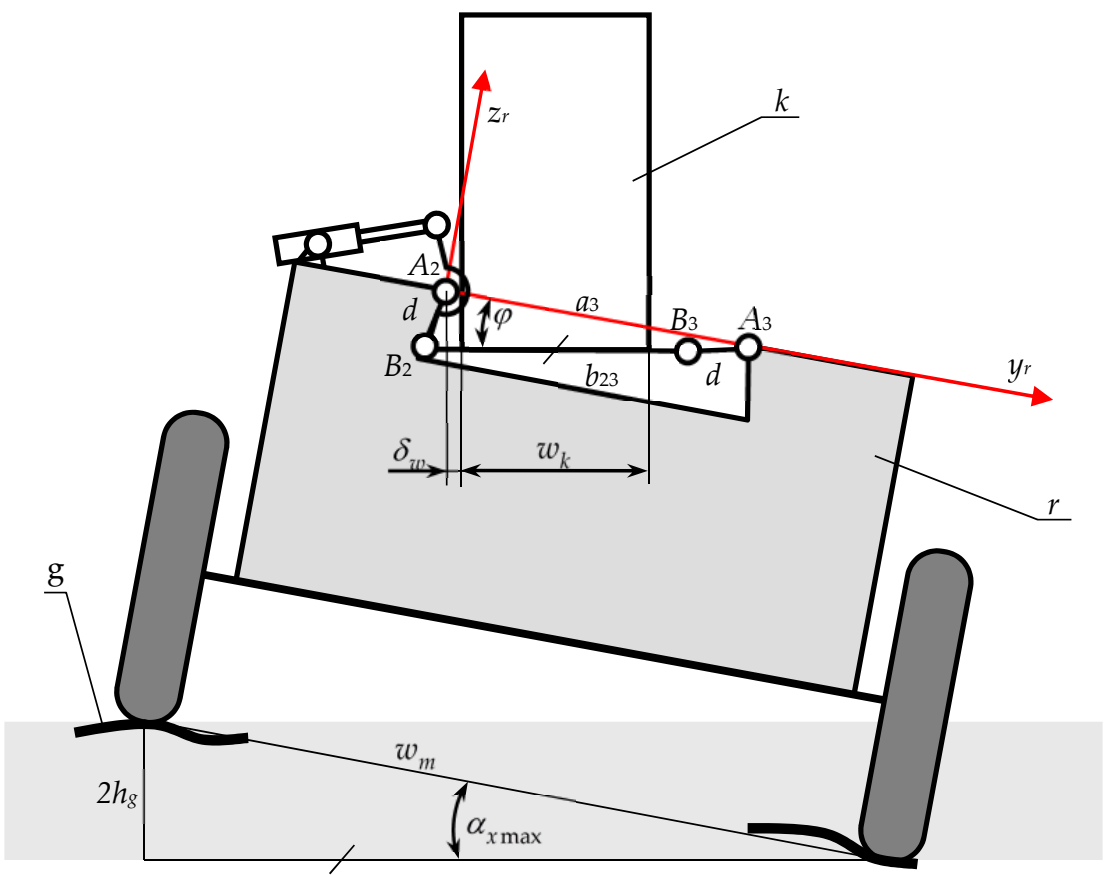

Figure 11. Frame tilt angle in relation to the singular position of the four bar linkage

Satisfying the inequality (84) quarantines a fail-safe operation of the four bar linkage $A_{2} A_{3} B_{3} B_{2}$ and of the cab. Conditions (82), (83), (84) yield the dimensions: $a_{3}, b_{2}, d$. When the machine is operated in uneven terrain where $2 h_{g}$ exceeds the predetermined value, the active suspension mechanism can reach the limits of its working field and the cab will momentarily deviate from the vertical direction.

\section{Simulation data of the active suspension system}

Machine specification data used in simulations:

$l_{m}=2.810[\mathrm{~m}]$ - distance between the front and rear axle of the machine frame, $w_{m}=1.980[\mathrm{~m}]$ - wheel spacing, $w_{r}=1.4[\mathrm{~m}]$ - frame width, $\mathbf{r}_{P_{r}}=[-2.1,-0.818,1.6][\mathrm{m}]$ position vector of the point $O_{r}, w_{k}=1.200[\mathrm{~m}]-$ cab width, ${ }^{p} r_{(p k) x}=0.000[m]$, ${ }^{p} r_{(p k) y}=0.000[m],{ }^{p} r_{(p k) z}=0.685[m]$ 
${ }^{p} r_{(p f) x}=0,000[m],{ }^{p} r_{(p f) y}=0,000[m], d f_{z}=0.335[m], m_{k}=480[k g]-$ cab mass, $m_{f}=160[k g]-$ mass of the seat with an operator, ${ }^{k} J_{k}=\left[\begin{array}{ccc}180 & 0 & 0 \\ 0 & 208 & 0 \\ 0 & 0 & 133\end{array}\right]\left[\mathrm{kg} \mathrm{m}^{2}\right]$ - inertia matrix of the cab in the reference system associated with the cab, ${ }^{f} J_{f}=\left[\begin{array}{ccc}23.8 & 0 & 0 \\ 0 & 24.7 & 0 \\ 0 & 0 & 13.2\end{array}\right]\left[\mathrm{kg} \mathrm{m}^{2}\right]$ - inertia matrix of the seat and operator in the system associated with the seat,

Road profile:

$2 h_{g}=0.250[\mathrm{~m}]$ - height of the unevenness range, $L_{g}=2[\mathrm{~m}]$ - wave length of the road unevenness, $\varphi=\pi / 2[\mathrm{rad}]$ - the phase shift angle between the left-and right-hand side of the machine, ${ }^{h} v_{P x, \max }=\frac{L_{g}}{2 \pi} \sqrt{\frac{g}{h_{g}}}=2.82[\mathrm{~m} / \mathrm{s}]$ maximal speed of the machine ride computed for the free wheel in contact with the road surface.

Active suspension mechanism for the cab:

$h_{m}=2.420[\mathrm{~m}]$ - distance of joints in the rocker arm connections $A_{2}$ and $A_{3}$ from the ground, $\delta_{w}=0.05[\mathrm{~m}]$ - admissible distance between the cab's side wall from the joint axis $A_{2}$ or $A_{3}$, $a_{3}=1.636[\mathrm{~m}], d=d_{3}=d=0.227[\mathrm{~m}], b_{23}=1.490[\mathrm{~m}], c_{2}=0.099[\mathrm{~m}], \quad \measuredangle\left(\mathbf{c}_{2}^{o}, \mathbf{d}_{2}^{o}\right)=4.5606[\mathrm{rad}]$ $a_{4}=0.541[\mathrm{~m}], \quad \mathbf{a}_{4}^{o}=[0.0000,0.2181,-0.9759][\mathrm{m}], \quad a_{1}=1.182[\mathrm{~m}]$, $\mathbf{a}_{1}^{o}=[-0.7218,0.6921,0.0000][\mathrm{m}], \quad b_{2}=b_{3}=0.5 b_{23}, \quad b_{1}=0.850[\mathrm{~m}], \quad s_{5 \min }=0.415[\mathrm{~m}]$, $s_{5 \max }=0.715[\mathrm{~m}], \dot{s}_{5 k \max }=1[\mathrm{~m} / \mathrm{s}], K_{5}=50[-]$.

$\mathbf{K}_{P}=\left[\begin{array}{ccc}20 & 0 & 0 \\ 0 & 20 & 0 \\ 0 & 0 & 0\end{array}\right][1 / s]-$ matrix of gain in the position path. The distance covered during the simulation $-10[\mathrm{~m}]$. The time step in the simulation procedure $-0.005[\mathrm{~s}]$.

Simulation data relating to the cab's and machine frame angular motion are given in Fig 12 and 13, each showing two plots of one angular velocity component in the function of time.

Simulation data relevant to the linear movement of the point $O_{f}$ on the seat are given in Fig 14 , showing the plots of vibration reduction factors for the three components of the rms acceleration derived from the formula:

$$
\mu_{l}=\sqrt{\int_{0}^{T} a_{f, o f f, l}^{2} d t / \int_{0}^{T_{s}} a_{f, o n, l}^{2} d t}
$$

where: $l=\left(x_{g}, y_{g}, z_{g}\right), a_{f, \text { off }, l}$ - linear acceleration in the direction $l$, the active suspension system being off, $a_{f, \text { on }, l}$ - linear acceleration in the direction $l$, the active suspension system being on; $T_{s}$ - simulation time associated with the ride velocity. 


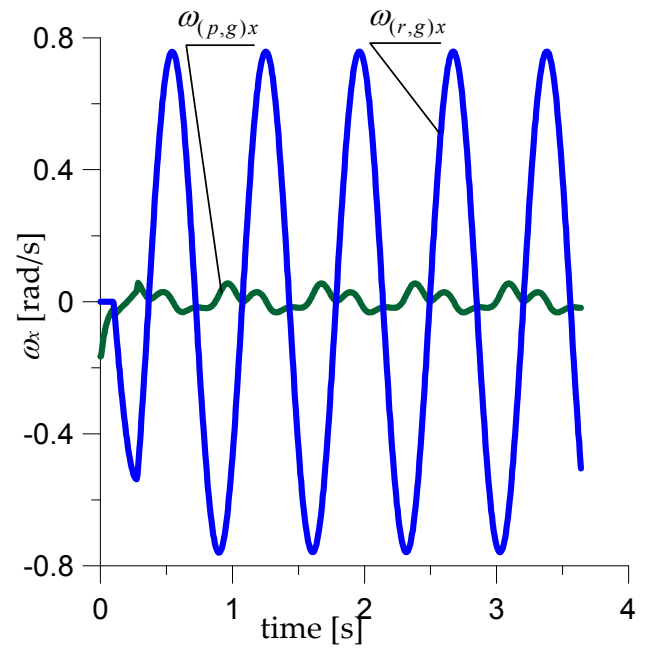

Figure 12. Coordinate $x_{r}$ of angular velocity of the frame and platform in the function of time, expressed in the system associated with the frame.

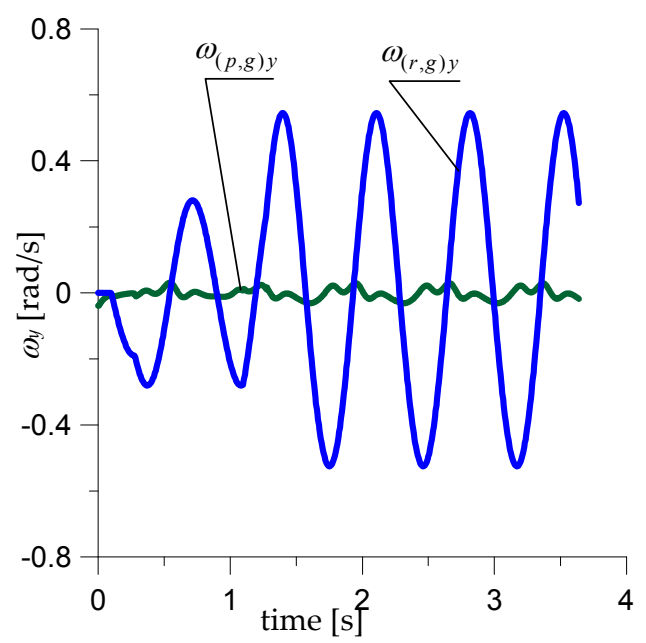

Figure 13. Coordinate $y_{r}$ of angular velocity of the frame and platform in the function of time, expressed in the system associated with the frame.

The mean power expended by the drives (shown in Fig 15) is derived from the formula:

$$
N_{\text {med }, j}=\frac{\int_{0}^{T_{s}} N_{j}^{+} d t}{T_{s}}
$$

where: $j=(1,4,5), N_{j}^{+}$- instantaneous positive power expended by the drive $j$. 


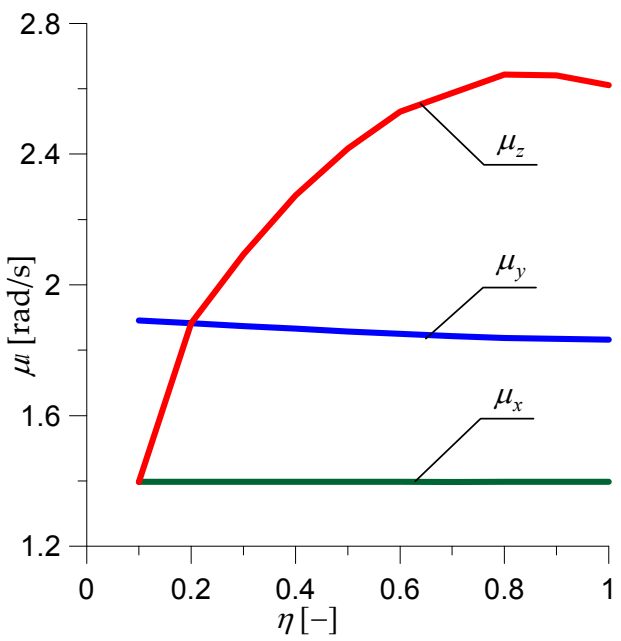

Figure 14. Vibration reduction of the point $O_{f}$ on the operator seat, in the directions $x_{r}, y_{r}, z_{r}$

Plots in Fig 14 and 15 show the relevant parameters in the function of the coefficient $\eta$ linearly related to the machine ride velocity, whilst for ${ }^{h} v_{P x}={ }^{h} v_{P x, \max }$ the value of $\eta$ becomes 1 .

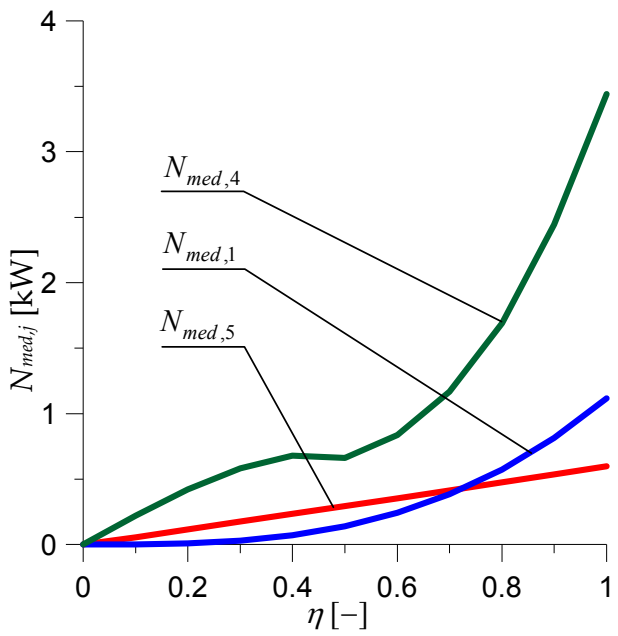

Figure 15. Mean power ratings of the drives 1, 4, 5

\section{Conclusions}

Simulations of the active suspension system performance have proved its adequacy in vibration reduction of angular vibrations of the cab around the longitudinal axis of the machine $x_{r}$ and around the transverse axis $y_{r}$. Seat vibrations along the vertical axis $z_{r}$ are successfully controlled, too. The applied procedure of dimension synthesis of the active 
suspension mechanism links leads to significant reduction of the cab and seat vibration in the direction $y_{r}$. Reduction of angular cab vibration around $y_{r}$ leads to reduction of linear seat vibrations in the direction $x_{r}$.

The operation of the active suspension system involves the real-time measurements of mechanical quantities which can be accurately measured with state-of-the-art sensors: angular velocity and acceleration of the frame, linear acceleration of a selected point on the machine frame, two angles of the frame tilting from the direction of the gravity forces and the length and velocities implemented by actuators.

Underlying the simulation procedure is that assumption that each computed drive velocity will be implemented without any time delay (provided that is allowed by collaborating programmes). Results therefore can be utilised when selecting drives which, when in extreme conditions, may not be able to perform the required movements. Besides, the overall time constant, taking into account the response time of the measurement system, the controls and drives becomes another limiting factor, particularly at higher frequencies of road input.

The actuator 4 handles two DOFs (i.e. the cab rotation around the $x_{r}$ axis and its translation along the $y_{r}$-axis) and induces slight movements of the cab in the direction of the $z_{r}$-axis, hence its power demand is higher than in drive 1.

\section{Author details}

Grzegorz Tora

Institute of Machine Design, Faculty of Mechanical Engineering, Cracow University of Technology, Cracow, Poland

\section{Acknowledgement}

I am particularly indebted to the Institute of Machine Design at Cracow Polytechnic for financial support needed to prepare this chapter and have it published in the book Vibration Control.

\section{References}

[1] Achen A, Toscano J, Marjoram R, StClair K, McMahon B, Goelz A, Shutto S (2008) Semiactive vehicle cab suspension using magnetorheological $(\mathrm{mr})$ technology. Proc. of the 7th JFPS Int. Symp. on Fluid Power, TOYAMA, 561-564.

[2] Jonasson M, Roos F (2008) Design and evaluation of an active electromechanical wheel suspension system, Mechatronics, 18: 218-230.

[3] Al Sayed B, Chatelet E, Baguet S, Jacquet-Richardet G (2011) Dissipated energy and boundary condition effects associated to dry friction on the dynamics of vibrating structures, Mechanism and Machine Theory 46: 479-491.

[4] Sampaio J V R (2009) Design of a Low Power Active Truck Cab Suspension, Eindhoven University of Technology, DCT № 119. 
[5] Du H, Lam J, Sze K Y (2003) Non-fragile output feedback $\mathrm{H}_{\infty}$ vehicle suspension control using genetic algorithm, Engineering Applications of Artificial Intelligence 16: 667-680.

[6] Duke M, Goss G, (2007) Investigation of Tractor Driver Seat Performance with Nonlinear Stiffness and On-off Damper, Biosystems Engineering 96 (4): 477-486.

[7] Jarviluoma M, Nevala K (1997) An Active Vibration Damping System of a Driver's Seat for Off-Road Vehicles, IEEE, 3: 38-43.

[8] Ruotsalainen P,Nevala K, Marjanen Y (2006) Design of an adjustable hydropneumatic damper for cab suspension, The XIII International Congress on Sound and Vibration, Vienna, July.

[9] Savkoor A, Manders S, Riva P (2001) Design of actively controlled aerodynamic devices for reducing pitch and heave of truck cabins, JSAE Review 22: 421-434.

[10] He Y, McPhee J (2005) Multidisciplinary design optimization of mechatronic vehicles with active suspensions, Journal of Sound and Vibration 283: 217-241.

[11] Yildirim S (2004) Vibration control of suspension systems using a proposed neural Network, Journal of Sound and Vibration 277: 1059-1069.

[12] Liu H, Nonami K, Hagiwara T (2008) Active following fuzzy output feedback sliding mode control of real-vehicle semi-active suspensions, Journal of Sound and Vibration 314: 39-52.

[13] Graf Ch, Maas J, Pflug H-Ch (2009) Concept for an Active Cabin Suspension, Proc. of the 2009 IEEE International Conference on Mechatronics. Malaga, Spain, April.

[14] Akcay H, Turkay S (2009) Influence of tire damping on mixed $H_{2} / H_{\infty}$ synthesis of half-car active suspensions, Journal of Sound and Vibration 322: 15-28.

[15] Koch G, Fritsch O, Lohmann B (2010) Potential of low bandwidth active suspension control with continuously variable damper, Control Engineering Practice 18: 1251-1262.

[16] Du H, Zhang N, Lam J (2008) Parameter-dependent input-delayed control of uncertain vehicle suspension, Journal of Sound and Vibration 317: 537-556.

[17] Marzbanrad J, Ahmadi G, Zohoor H, Hojjat Y (2004) Stochastic optimal previewcontrol of a vehicle suspension, Journal of Sound and Vibration 275, 973-990.

[18] Dasgupta B, Choudhury P (1999) A general strategy based on the Newton-Euler approach for the dynamic formulation of parallel manipulators, Mechanism and Machine Theory 34: 801-824.

[19] Tora G (2008) Kinematyka mechanizmu platformowego w układzie aktywnej redukcji drgań, XXI Ogólnopolska Konferencja Naukowo-Dydaktyczna Teorii Maszyn i Mechanizmów, Wydawnictwo ATH w Bielsku Białej ISBN 978-83-60714-57-7: 357-366. 